\title{
[O I] sub-arcsecond study of a microjet from an intermediate mass young star: RY Tauri`
}

\author{
V. Agra-Amboage ${ }^{1}$, C. Dougados ${ }^{1}$, S. Cabrit ${ }^{2}$, P. J. V. Garcia ${ }^{3,4,1}$, and P. Ferruit ${ }^{5}$ \\ 1 Laboratoire d'Astrophysique de l'Observatoire de Grenoble, UMR 5521 du CNRS, 38041 Grenoble Cedex, France \\ e-mail: [amboage; dougados]@obs.ujf-grenoble.fr \\ 2 LERMA, Observatoire de Paris, UMR 8112 du CNRS, 61 avenue de l'Observatoire, 75014 Paris, France \\ 3 Departamento de Engenharia Fisica, Faculdade de Engenharia, Universidade do Porto 4200-465 Porto, Portugal \\ 4 Centro de Astrofisica, Universidade do Porto, 4150-752 Porto, Portugal \\ 5 Université de Lyon, 69003 Lyon; Université de Lyon-1, Observatoire de Lyon, 9 Av. Charles André, 69230 St. Genis Laval; \\ CNRS, UMR 5574, Centre de Recherche d'Astrophysique de Lyon; École Normale Supérieure de Lyon, 69007 Lyon, France
}

Received 22 April 2008 / Accepted 21 October 2008

\begin{abstract}
Context. High-resolution studies of microjets in T Tauri stars (cTTs) reveal key information on the jet collimation and launching mechanism, but only a handful of systems have been mapped so far.

Aims. We wish to perform a detailed study of the microjet from the $2 M_{\odot}$ young star RY Tau, to investigate the influence of its higher stellar mass and claimed close binarity on jet properties.

Methods. Spectro-imaging observations of RY Tau were obtained in [O I] $\lambda 6300$ with resolutions of $0 !^{\prime} 4$ and $135 \mathrm{~km} \mathrm{~s}^{-1}$, using the integral field spectrograph OASIS at the Canada-France-Hawaii Telescope. Deconvolved images reach a resolution of 0.'2.

Results. The blueshifted jet is detected within $2^{\prime \prime}$ of the central star. We determine its PA, collimation, 2D kinematics, mass-flux rate, ejection to accretion ratio, and transverse velocity shifts taking accurately into account errors due to finite signal to noise ratio. The RY Tau system is shown to provide important constraints to several models of steady MHD ejection.

Conclusions. The remarkably similar properties of the RY Tau microjet compared to jets from lower mass cTTs gives support to the common belief that the jet launching mechanism is universal over a broad range of stellar masses. The proximity between the jet PA and the PA of the photocenter variations observed by Hipparcos calls into question the interpretation of the latter in terms of binarity of RY Tau. Partial occultation events of the photosphere may offer an alternative explanation.
\end{abstract}

Key words. ISM: jets and outflows - ISM: individual objects: HH 938 - stars: formation - stars: individual: RY Tau stars: winds, outflows

\section{Introduction}

One of the main open problems in star formation is to understand the physical mechanism by which mass in young stars is ejected from the accreting system and then collimated into jets. Magneto-hydrodynamic accretion-driven wind models best explain the efficient collimation and the large mass ejection efficiencies observed. However, different scenarios are proposed for the origin of the outflow, depending on whether it originates from the stellar surface (Sauty \& Tsinganos 1994; Matt \& Pudritz 2008), from the inner edge of the accretion disk (Shu et al. 1995), an extended range of disk radii (Pudritz \& Norman 1986; Casse \& Ferreira 2000), or from reconnexion sites in the stellar magnetosphere (Goodson et al. 1997; Ferreira et al. 2000).

Microjets from $\mathrm{T}$ Tauri stars offer a unique opportunity to probe the inner 100 AUs of the outflow where the acceleration and collimation processes occur, and therefore to place strong observational constraints on the ejection mechanism in young

* Based on observations obtained at the Canada-France-Hawaii Telescope (CFHT) which is operated by the National Research Council of Canada, the Institut National des Sciences de l'Univers of the Centre National de la Recherche Scientifique of France, and University of Hawaii. stars. Combined kinematic/imaging studies of microjets at subarcsecond resolution have allowed major advances in this field in recent years (Lavalley et al. 1997; Lavalley-Fouquet et al. 2000; Bacciotti et al. 2000; Ray et al. 2007, and refs. therein). That the jet phenomenon is very robust over orders of magnitude of central stellar mass is testified by the fact that both brown dwarfs and Herbig Ae/Be stars are known to drive collimated outflows (Whelan et al. 2005; Corcoran \& Ray 1997). However, variation of jet properties (collimation, kinematics, mass-loss rates) with mass of the driving source has not yet been investigated in detail. It is thus important to extend the sample of microjets studied at high angular resolution to central sources of differing mass and binary status.

We concentrate here on the intermediate-mass classical T Tauri star RY Tau, located in the nearby Taurus-Auriga cloud $\left(d=140 \mathrm{pc}\right.$ ). With spectral type F8-G1 and $M_{\star} \sim 2 M_{\odot}$ (Mora et al. 2001; Calvet et al. 2004), RY Tau is of significantly higher mass than other nearby atomic jet sources previously spectroimaged at high resolution, the most massive so far being RW Aur with $1.4 M_{\odot}$ (Woitas et al. 2001). It thus allows us to probe jet formation in a mass range intermediate between standard classical $\mathrm{T}$ Tauri stars and Herbig Ae/Be stars (of mass $>2 M_{\odot}$ ). The presence of a jet in RY Tau was indirectly suggested by 
[O I] emission blueshifted by $-70 \mathrm{~km} \mathrm{~s}^{-1}$ in its spectrum (Cabrit et al. 1990; Hartigan et al. 1995). It has been recently confirmed by St-Onge \& Bastien (2008) who detected a collimated string of $\mathrm{H} \alpha$ emission knots (HH 938) extending from 1.5" out to several arcminutes on both sides of the star. RY Tau is also an active accretor, with veiling values of $\sim 0.1$ in the optical (Hartigan et al. 1995; Basri et al. 1991) and 0.8 in the UV (Calvet et al. 2004). The latter corresponds to an updated accretion rate of 6.4-9.1 $\times 10^{-8} M_{\odot} \mathrm{yr}^{-1}$, 4 times higher than the previous determination by Hartigan et al. (1995).

Another important peculiarity of RY Tau besides its mass is a suspected close binary status from Hipparcos observations. The variability of the astrometric solution, indicating motion of the photocentre, is interpreted as indicative of a binary of PA = $304^{\circ} \pm 34^{\circ}$ and minimum separation of 3.27 AU (Bertout et al. 1999). Such a close binary companion might have a strong impact on the ability of the inner disc regions to drive a collimated outflow, possibly leading to observable differences to microjets from single stars.

In addition to these specific properties, RY Tau shows a remarkably flat spectral energy distribution in the far-infrared, and a rather large degree of linear polarisation of a few percent in the optical, indicating a substantial amount of circumstellar material (Bastien 1982). RY Tau also shows a peculiar photometric variability with large variations of brightness accompanied by a near constancy of colour. Two abrupt brightnening events were recorded in 1983/1984 and 1996/1997 reminiscent of UX Ori events (Herbst \& Stine 1984; Petrov et al. 1999). It is also a rapid rotator with a $v \sin i$ of $52 \pm 2 \mathrm{~km} \mathrm{~s}^{-1}$ (Petrov et al. 1999). Petrov et al. (1999) argue that the photometric behavior can be interpreted as variable obscuration of the central star by a disc seen at high inclination. The large values of $v \sin i$ and polarisation further support this conclusion.

We present in this paper sub-arcsecond optical spectroimaging observations of the RY Tau microjet in [O I] obtained with the OASIS integral field spectrograph coupled with adaptive optics correction at the Canada France Hawaii telescope. The combination of high angular resolution and intermediate spectral resolution allows for accurate subtraction of the strong central continuum emission, critical to study the inner regions of the jet. Details on the observations and data reduction are given in Sect. 2. In Sect. 3, we discuss the main results regarding the jet morphology, the jet kinematics both along and transverse to the jet axis, the search for rotation signatures and the derivation of mass-loss rates. We analyze these results in the context of previous studies of microjets and discuss their implication for jet launching models and binarity of RY Tau in Sect. 4. We conclude in Sect. 5.

\section{Observations and data reduction}

Observations of the RY Tau microjet were conducted on January 15th 2002 at the Canada France Hawaii Telescope (CFHT), using the integral field spectrograph OASIS combined with the adaptive optics system PUE'O. The configuration used for the RY Tau observations provides a spectral resolution of $R \sim 3000$ (velocity resolution $F W H M=135 \mathrm{~km} \mathrm{~s}^{-1}$ estimated from the width of the [O I] telluric emission line) and a velocity sampling of $41 \mathrm{~km} \mathrm{~s}^{-1}$ over a spectral range from $6209 \AA$ to $6549 \AA$, including the [O I] $6300 \AA$ line. The field of view is $6.2^{\prime \prime} \times 5.0^{\prime \prime}$ with a spatial sampling of $0.16^{\prime \prime}$ per lenslet. After AO correction, the effective spatial resolution achieved is $0 .{ }^{\prime} 4$ (Gaussian core $F W H M$ ). One exposure with an on-source integration time of $1800 \mathrm{~s}$ was obtained.

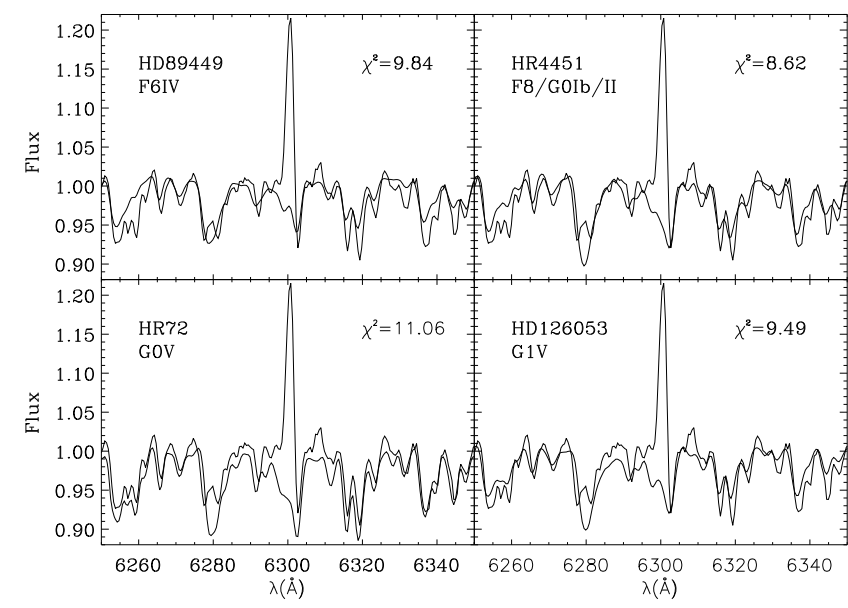

Fig. 1. Comparison between the EXPORT RY Tau spectrum (thick line, from Mora et al. 2001) and rotationally broadened EXPORT spectra of four reference stars (thin lines). The reduced $\chi^{2}$, computed over our 6210-6550 $\AA$ wavelength domain (excluding the [O I] line region) is indicated in each panel. The reference star that best fits the photospheric spectrum of RY Tau is HR 4451.

The data reduction was carried out following the standard OASIS procedure (Bacon et al. 2001), using the XOasis software. A dedicated spectral extraction procedure was developed for the OASIS January 2002 run, to correct for a slight rotation of the lenslet array. Spectro-photometric calibration was performed using the standard star HD 93521. Final spectra are calibrated in units of $10^{-19} \mathrm{~W} \mathrm{~m}^{-2} \AA^{-1}\left({ }^{\prime \prime}\right)^{-2}$. Atmospheric refraction correction was performed a-posteriori by recentering each spectral image on the continuum centroid, following Garcia et al. (1999). Subsequent analysis of the data (continuum subtraction, removal of [O I] sky emission, construction of images and PV diagrams) was performed under IDL.

As Fig. 1 illustrates, the red wing of the [O I] line in RY Tau is strongly distorted by an underlying photospheric absorption line. Therefore, the photospheric spectrum of RY Tau has to be carefully subtracted in order to retrieve the intrinsic jet kinematics and flux close to the source.

Since we did not observe a standard star of similar spectral type as RY Tau with OASIS, we retrieved from the litterature medium resolution $(R=6600)$ optical spectra of both RY Tau and standard stars obtained in the context of the EXPORT project, published in Mora et al. (2001). We investigated four different reference stars: HD 126053 (G1V), HD 89449 (F6IV), HR 4451 (F8/G0Ib/II) and HR 72 (G0V) to find the best fit to the continuum. Figure 1 shows the comparison between the EXPORT spectrum of RY Tau and the photospheric contribution predicted by the four stars, after rotational broadening by $v \sin i$ $=52 \mathrm{~km} \mathrm{~s}^{-1}$. In accordance with Mora et al. (2001), we find that the best fit is obtained for HR 4451, of spectral type F8/G0.

We then developed a dedicated continuum subtraction procedure under IDL in order to remove the photospheric contribution from our OASIS [O I] $\lambda 6300 \AA$ spectra at distances $d<1^{\prime \prime}$ from the star. The EXPORT spectrum of HR 4451 is first rotationally broadened to the $v \sin i$ of RY Tau and smoothed to the OASIS spectral resolution. At each lenslet position, this standard spectrum is scaled to the continuum level in the current OASIS spectrum, as illustrated in the top-left panel of Fig. 2. It is then subtracted out, leaving a residual [O I] $\lambda 6300 \AA$ line profile essentially free of photospheric features, shown by the solid curve in the bottom panel of Fig. 2. The good photospheric subtraction 

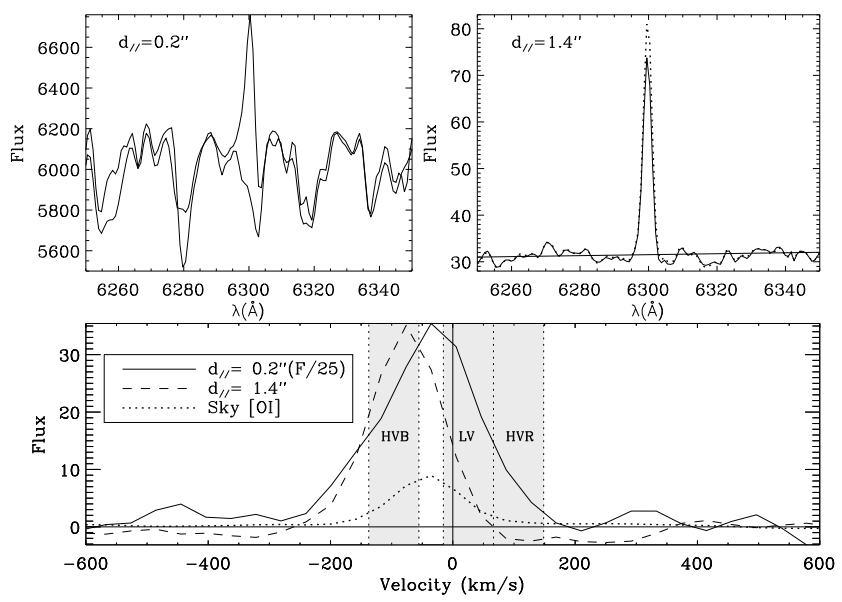

Fig. 2. Top panels: solid curves show the observed OASIS [O I] $\lambda 6300 \AA$ spectra (thick lines) and the fitted continuum (thin lines) towards the blueshifted jet, offset by 0.'2 (top-left) and 1.'4 (top-right) from the star. Profiles before subtraction of the [O I] atmospheric line are shown with a dotted line (same as solid thick curve at 0.2 ). Bottom panel: residual continuum-subtracted [O I] line profiles at 0.2 and 1.' 4 from the star. The subtracted [OI] atmospheric line is shown as a dotted curve. The profile at 0.2 is divided by 25 for clarity. Velocity intervals contributing to the HVB, LV and HVR channel maps of Fig. 3 are shaded.

indicates no detectable veiling in our OASIS spectra, consistent with the low veiling $\simeq 0.1$ in previous high-resolution optical spectra of RY Tau (Hartigan et al. 1995; Basri et al. 1991). At larger distances $d \geq 1^{\prime \prime}$, photospheric absorption lines no longer contribute significantly, and a simple linear baseline fit to the local continuum over two intervals on either side of the [O I] line is used, as illustrated in the top-right panel of Fig. 2. In each residual, continuum-subtracted spectrum, we also estimate the spectral noise $\sigma$ equal to the standard deviation computed over two wavelength intervals bracketting the [O I] line. This noise estimate thus takes into account both the original data noise and the uncertainty in the photospheric continuum subtraction.

The residual [O I] line emission, especially in the red wing and close to the source, will depend strongly on the estimate of the photospheric line lying underneath. The depth of this absorption feature is seen to vary with the spectral type and/or the luminosity class. However, from the higher resolution spectra obtained by Mora et al. (2001), we see that the depth of the absorption line is well matched by the photospheric spectrum of HR 4451 (Fig. 1). We thus feel confident that our photospheric fitting procedure does not overestimate the red wing of the $[\mathrm{OI}]$ line emission close to the source.

[O I] atmospheric line emission is estimated from the average of 13 spectra located at the periphery of the field of view $\left(\alpha_{\mathrm{c}}=2^{\prime \prime}, \delta_{\mathrm{c}}=-1^{\prime \prime}\right)$. It is then subtracted from each spectrum. This average sky profile is shown with the dotted line in the bottom panel of Fig. 2. The average peak radial velocity is $-41 \mathrm{~km} \mathrm{~s}^{-1}$ with respect to RY Tau and the average peak surface brightness is $9.6 \times 10^{-19} \mathrm{~W} \mathrm{~m}^{-2} \AA^{-1}\left({ }^{\prime \prime}\right)^{-2}$. We derive a velocity resolution of $135 \mathrm{~km} \mathrm{~s}^{-1}$ from the $F W H M$ of the sky [O I] line profile. From the lens-to-lens dispersion in the [O I] sky line centroid velocities (estimated through Gaussian fitting), we derive a random uncertainty in the velocity calibration of $5 \mathrm{~km} \mathrm{~s}^{-1}$ (rms). The wavelength scale is converted to a radial velocity scale with respect to the central source, using a heliocentric radial velocity for RY Tau of $V_{\text {helio }}=18 \mathrm{~km} \mathrm{~s}^{-1}$ (Petrov et al. 1999).

\section{Results}

\subsection{Morphology}

\subsubsection{Raw $\left[\mathrm{O}_{1}\right]$ channel maps}

Line emission maps in various velocity intervals are reconstructed by reprojecting the hexagonal OASIS lenslet array onto a square grid with 0 '. $^{\prime} 1$ sampling. In the top row of Fig. 3, we display continuum-subtracted line emission maps in three velocity intervals, each covering two individual spectral channels of width $41 \mathrm{~km} \mathrm{~s}^{-1}$ : high-velocity blue (HVB): [-137.5: -55.5] $\mathrm{km} \mathrm{s}^{-1}$, low-velocity (LV): [-15.5: +66.5] $\mathrm{km} \mathrm{s}^{-1}$, high-velocity red (HVR): [+66.5: $+148.5] \mathrm{km} \mathrm{s}^{-1}$. These intervals are shaded in grey over the profiles in Fig. 2. The channel centered at $-35 \mathrm{~km} \mathrm{~s}^{-1}$ shows a behavior intermediate between the HVB and LV intervals. It is thus left out from the channel maps, in order to better reveal the distinct morphologies between the high and low velocity ranges.

The continuum map, computed by integration of the estimated photospheric contribution over the velocity interval $\left[-2400,-500 \mathrm{~km} \mathrm{~s}^{-1}\right]$, is also shown in the last top panel. Fitting the brightness radial distribution of the continuum map with a Moffat function, representative of a partially corrected $\mathrm{AO}$ point-spread function (PSF), gives a Gaussian core width of $F W H M=0 . ' 4$. We determine the centroid continuum position with an accuracy $(1 \sigma)$ of $0{ }^{\prime} 015$ from 2D Gaussian fitting. In all figures, spatial offsets are plotted relative to this continuum centroid.

In the HVB map, tracing high blueshifted velocities, the [O I] jet emission is clearly detected out to distances of $2^{\prime \prime}$ towards the north-west. The line emission peak is slightly displaced along the jet from the continuum centroid position $(\Delta \alpha=$ $\left.-0{ }^{\prime} 05, \Delta \delta=0{ }^{\prime} 035\right)$ and the emission is resolved $(F W H M=$ 0 .'5). In the LV map, tracing low flow velocities, the emission is marginally resolved $\left(F W H M=0{ }^{\prime} \cdot 45\right)$ and centered closer to the continuum position $\left(\Delta \alpha=-0^{\prime}{ }^{\prime} 032, \Delta \delta=00^{\prime} 018\right)$. At high redshifted velocities (HVR map), the emission is dominated by an unresolved component $(F W H M=0 . ' 4)$ centered on the continuum position within positional uncertainties $\left(\Delta \alpha=-0{ }^{\prime} 025\right.$, $\left.\Delta \delta=-0 .{ }^{\prime} 016\right)$. Some low level extended emission also appears towards the east. This extension is also faintly present in the LV map, although its relative contribution is much less important. We checked that this residual redshifted emission remains when using another reference star for the photospheric emission subtraction. This extended component shows a position angle significantly different from the one of the blueshifted jet emission and might trace strong brightness asymmetry in the counterjet emission. However this feature has a low signal to noise ratio (between 3 and 5, see Fig. 3), thus preventing us from a detailed analysis of its possible origin.

\subsubsection{Deconvolved channel maps}

In order to remove the non Gaussian wings of the partially corrected AO PSF and derive accurate estimates of jet position angle and jet emission widths, we have deconvolved the observed channel maps, using the continuum map as an estimate of the corresponding point spread function. We use the LUCY restoration routine as implemented in the STSDAS/IRAF package. We limit ourselves to 20-25 iterations (standard acceleration method) which yield $\chi^{2}$ values of $1.4,1.1,2.0$ for the 3 channels respectively and final resolution of $F W H M=0.2$ (estimated by fitting a Gaussian profile to the central compact component in the HVR map). The maximum number of 


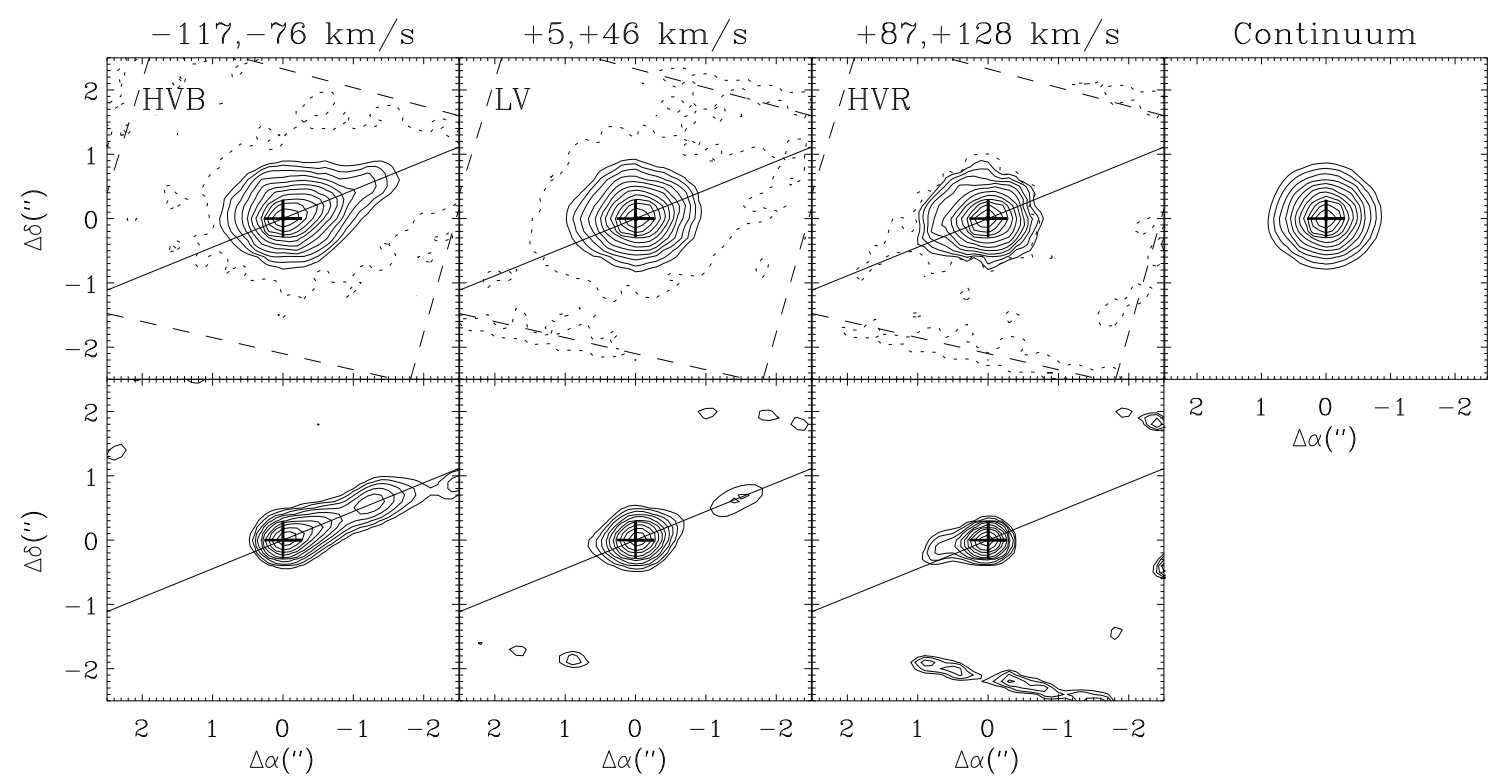

Fig. 3. Continuum-subtracted [OI] $\lambda 6300 \AA$ A emission maps of the RY Tau microjet, both raw (top panels) and deconvolved (bottom panels). Emission is integrated over three velocity intervals, with the centers of the corresponding velocity channels (each $41 \mathrm{~km} \mathrm{~s}^{-1}$ wide) indicated on top. The derived blueshifted jet position angle is plotted as a solid line. The continuum map is displayed in the top-right panel. In the top row, contours start at $2 \%$ of the maximum and increase by factors of $\sqrt{2}$. In the bottom row they start at $0.1 \%$ of the maximum and increase by factors of 2. Dotted contours correspond to a signal to noise ratio of 5 for the HVB and LV maps, 3 for the HVR map. The dashed line shows the OASIS field of view. Spatial offsets are relative to the continuum centroid (located with a cross). The spatial sampling used is 0 .' $^{\prime} 1$.

iterations was determined by ensuring that the derived image characteristics (such as intrinsic jet $F W H M$ ) did not change significantly with further iterations. The deconvolved maps are shown in the bottom panels of Fig. 3.

As already noticed in the raw maps, the line emission is dominated in all channels by a compact component very close to the star. It is unresolved and centered at the continuum position in the HVR map, and shows increasing FWHM and spatial offset towards more blueshifted velocities. The extended jet emission stands out more clearly in the deconvolved HVB map, with the low-level emission in the raw map sharpening into an emission knot located at $\Delta \alpha=-1$ '.23, $\Delta \delta=0.55$, ie at a distance of $1 . .35$ (190 AU) from the star. In the HVR map, the low level extension towards the east stands out clearly (at a level of $0.2 \%$ of the peak emission).

From the HVB deconvolved map, we derive a position angle (PA) for the blueshifted jet of $294^{\circ} \pm 1^{\circ}$. This orientation agrees with the mean of the PA values of $292^{\circ}-297^{\circ}$ derived on larger scales for $\mathrm{H} \alpha$ knots by St-Onge \& Bastien (2008). Our derived blueshifted jet PA is perpendicular to the direction of the velocity gradient in interferometric millimetric CO maps or RY Tau, $\mathrm{PA} \simeq 21^{\circ}$ (Koerner \& Sargent 1995), indicating that the jet is parallel to the spin axis of the disk. It is also perpendicular to the average direction of the optical linear polarization vector derived by Bastien (1982) of $20^{\circ}$. Interestingly, the derived jet PA is also compatible with the direction of the photocenter variation seen by Hipparcos $\left(\mathrm{PA}=304^{\circ} \pm 34^{\circ}\right)$, interpreted as the direction to a possible binary companion (Bertout et al. 1999). We will return to this issue in the discussion section.

\subsubsection{Jet widths}

We show in Fig. 4 the variation of the intrinsic jet width as a function of the distance to the star. We estimate the intrinsic jet

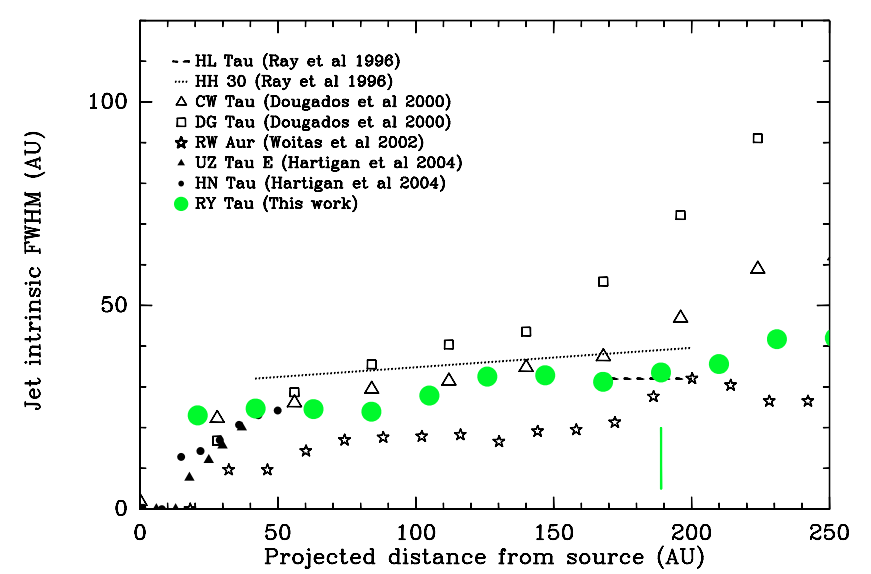

Fig. 4. Large filled circles: PSF-corrected width $(F W H M)$ of the RY Tau jet forbidden line emission versus projected distance from the star, as derived from the [O I] HVB deconvolved map (see text). Measurements available on the same spatial scales for other TTS microjets are also plotted with various symbols, after correction for the corresponding PSF. The object-symbol correspondance and references are given at the top left corner of the plot. The position of the knot located at 1'.35 = $190 \mathrm{AU}$ in the RY Tau microjet is denoted by the vertical line.

width as: $F W H M_{0}=\sqrt{F W H M^{2}-(0 . ' 2)^{2}}$, where $F W H M$ is measured from Gaussian fits to the 1D transverse jet emission profiles in the deconvolved HVB map, of effective resolution 0.'2. The RY Tau jet width increases slowly from 0.2 (28 AU) at projected distances 0 .' $^{\prime} 15<d<00^{\prime} 6(20-84 \mathrm{AU})$ to 0. '3 (42 AU) at $d=2^{\prime \prime}(280 \mathrm{AU})$ with a full opening angle of $\sim 5^{\circ}$. This behavior is remarkably similar to that of other $\mathrm{T}$ Tauri microjets observed at sub-arcsecond resolution (see Fig. 4 and discussion section). We do not see any clear change of jet width at the location of the knot (projected distance along the jet of $190 \mathrm{AU}$ ), within our angular resolution. 

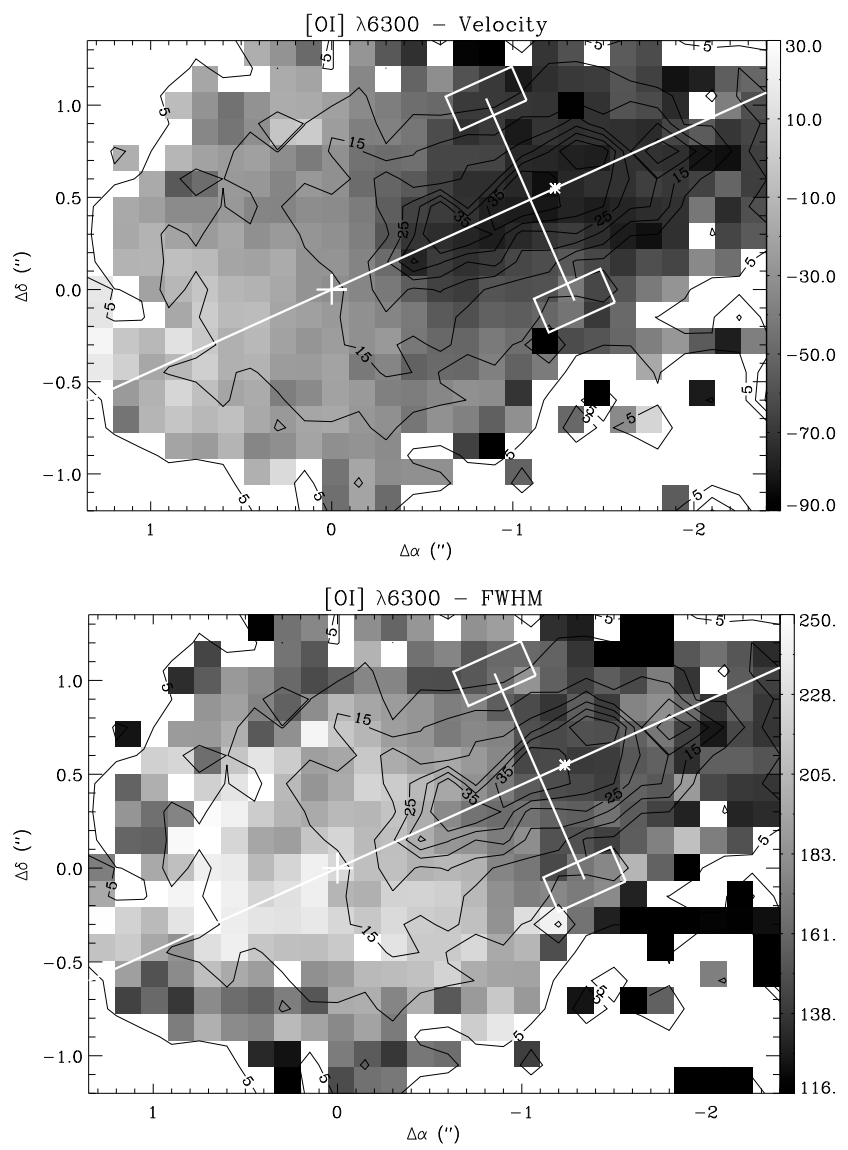

Fig. 5. Maps of the centroid radial velocity (top) and velocity width (bottom) of a one-component Gaussian fit to the [OI] $\lambda 6300 \AA$ line profiles. Contours show the signal to noise ratio $(S N R)$ at the line peak (from 5 to 35 by step of 5). Only lenslets with $S N R>5$ are shown. The cross shows the continuum position, the asterisk denotes the HVB knot, and the straight line the blue jet PA. The two white boxes indicate the two regions that contribute to the transverse velocity shift detected at 1.'2 from the star and 0.' 6 to the jet axis in Fig. 8.

\subsection{Kinematics}

\subsubsection{Centroid velocity and line width maps}

High spectral resolution integrated line profiles of $[\mathrm{OI}]$ in RY Tau show two distinct kinematical components, a high-velocity component (HVC) with peak radial velocities of $-70,-80 \mathrm{~km} \mathrm{~s}^{-1}$ and a low-velocity component (LVC) peaked at $-4 \mathrm{~km} \mathrm{~s}^{-1}$ (Hartigan et al. 1995; Hirth et al. 1997). Since both components contribute close to the star, we first tried to perform two-component Gaussian fits to each individual OASIS line profile. However, because of insufficient spectral resolution, the two velocity components cannot be well separated in our data (see bottom panel of Fig. 2) and a globally consistent fit could not be found over the whole field of view.

To obtain an overall view of the 2D kinematics of the RY Tau jet, we thus performed a single-component Gaussian fit to our spectra. We show in Fig. 5 the resulting maps of centroid velocity and profile $F W H M$. We overlay on these maps the contours of the signal to noise ratio $(S N R)$ at the line peak. Note that due to combined strong photon noise and strong residuals of photospheric subtraction close to the central source, the maximum peak $S N R$ is not reached at the star position but towards the blueshifted jet. In photon noise statistics, and for an infinite sampling, the standard deviation of the centroid estimate

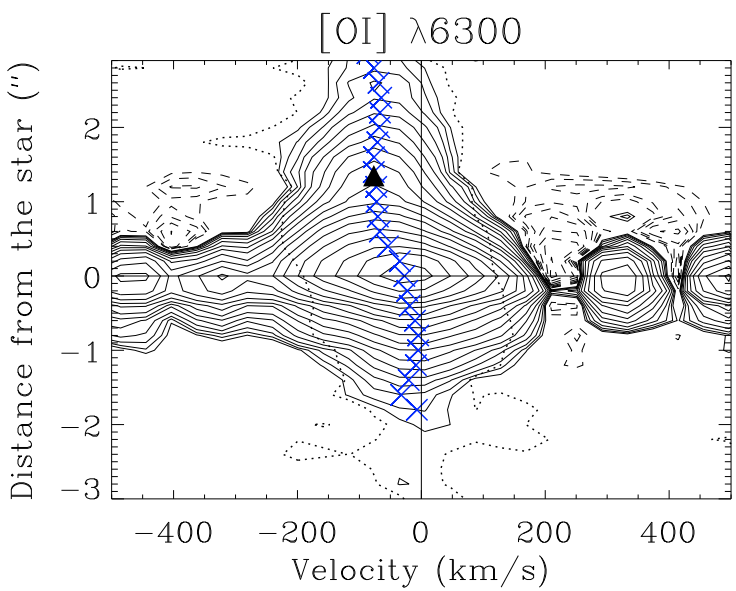

Fig. 6. Position-velocity map along the RY Tau jet in [O I] $] 6300 \AA$ after continuum subtraction, for a $1^{\prime \prime}$ wide pseudo-slit sampled every 0 '.2 along the jet. Centroid velocities derived from a single Gaussian fit are also plotted (blue crosses). Intensity contours start at $3 \sigma(1.32 \times$ $\left.10^{-19} \mathrm{~W} \mathrm{~m}^{-2} \AA^{-1}\left({ }^{\prime \prime}\right)^{-2}\right)$ and increase by factors of $\sqrt{2}$. Negative contours are shown with dashed lines. The dotted line shows the $5 \sigma$ level, based on the spectral noise in each spectrum. Close to the star, the far wings of the line $\left(|v| \geq 250 \mathrm{~km} \mathrm{~s}^{-1}\right)$ are affected by continuum subtraction residuals. The location of the jet knot identified in the deconvolved HVB map is indicated by a filled triangle.

for a Gaussian distribution of rms standard deviation $\sigma_{\text {line }}$ and peak signal-to-noise $S N R$ is given by: $\sigma_{\text {cent }}=\frac{\sigma_{\text {line }}}{S N R}$ (Porter et al. 2004). Uncertainties in line centroid velocities derived from our Gaussian fitting procedure are thus typically: $\sigma_{V}=\frac{F W H M / 2.35}{S N R}$, i.e. $\leq 11-16 \mathrm{~km} \mathrm{~s}^{-1}$ for a line peak $S N R \geq 5$ and a line $F W H M$ ranging from 135 to $200 \mathrm{~km} \mathrm{~s}^{-1}$ typical of our observations.

The centroid map shows that the extended blueshifted jet emission at $\mathrm{PA}=294^{\circ}$ has centroid velocities of average value $-70 \mathrm{~km} \mathrm{~s}^{-1}$, consistent with the HVC in previous high-spectral resolution observations. The jet shows a local peak in centroid velocity $\left(-90 \mathrm{~km} \mathrm{~s}^{-1}\right)$ at $(\Delta \alpha, \Delta \delta) \sim\left(-1^{\prime \prime} .2,+0^{\prime} .6\right)$, coincident with the emission knot identified in the deconvolved HVB channel map (denoted with an asterisk in Fig. 5). The profile width is narrow, and essentially unresolved beyond 1'. 3 from the star.

Closer to the star, centroid velocities progressively decrease to values of $\simeq-30,-10 \mathrm{~km} \mathrm{~s}^{-1}$ while the line $F W H M$ significantly increases. The available velocity information combined with the channel maps from Fig. 3 suggests that, towards the central source, emission from the spatially unresolved LVC component at $-4 \mathrm{~km} \mathrm{~s}^{-1}$ centered at the continuum position strongly contributes to the line profile, resulting in intermediate centroid velocities and increased line widths.

In a small zone on the redshifted side, at $(\Delta \alpha, \Delta \delta) \sim$ $(+0.8,-0 ! 3)$, peak velocities appear slightly redshifted and line profiles become significantly broader with a fitted $F W H M \geq$ $200 \mathrm{~km} \mathrm{~s}^{-1}$, corresponding to an intrinsic $F W H M>140 \mathrm{~km} \mathrm{~s}^{-1}$, suggesting a contribution from a third kinematical component. This region spatially coincides with the red extension identified in the reconstructed HVR channel map from Fig. 3.

\subsubsection{Kinematics along the jet axis}

Figure 6 shows a position-velocity map along the jet in [OI], reconstructed by averaging the lenslets transversally across a $1^{\prime \prime}$ wide pseudo-slit, and sampling every 0.2 along the jet. Centroid velocities derived from one-component Gaussian fits to the line profiles are also plotted. Beyond 0.'5, we clearly 


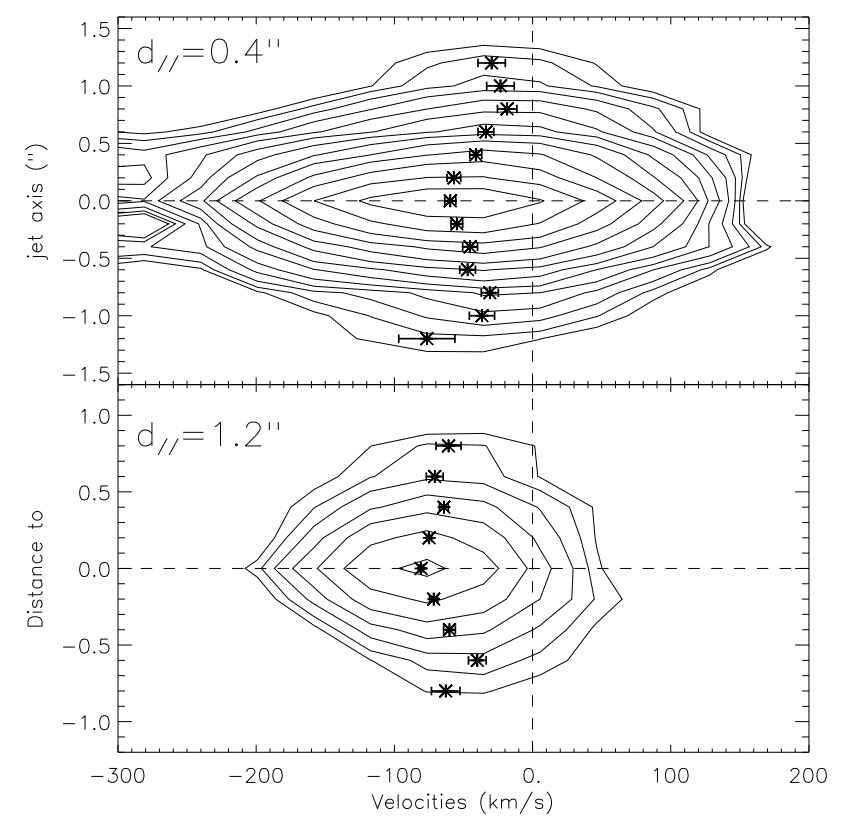

Fig. 7. Transverse position-velocity diagrams across the blue jet at distances of 0.4 (top) and 1'.2 (bottom) from the star. Centroid velocities (crosses) derived from one-component Gaussian fits are also plotted with their estimated $1 \sigma$ error bars (see text). The pseudo-slits are 0.2 wide and are binned every 0.' 2 in the transverse direction. Contours start at $5 \sigma$ and increase by factors of $\sqrt{2}$.

detect the high-velocity blue jet (HVC) with an average centroid radial velocity of $\sim-70 \mathrm{~km} \mathrm{~s}^{-1}$. The profiles are essentially unresolved spectrally in the jet $\left(F W H M=140 \mathrm{~km} \mathrm{~s}^{-1}\right)$. Average variations in radial velocities along the blue jet do not exceed $10 \%\left(7 \mathrm{~km} \mathrm{~s}^{-1}\right)$ beyond 0.6 over the central $2 . .5$. Closer to the star, centroid radial velocities progressively decrease and converge to $\sim-10 \mathrm{~km} \mathrm{~s}^{-1}$ on the redshifted side, consistent with the LVC. The lines become asymmetric and wider with an intrinsic FWHM (deconvolved from the instrumental $F W H M=135 \mathrm{~km} \mathrm{~s}^{-1}$ ) of $\simeq 130 \mathrm{~km} \mathrm{~s}^{-1}$ (see profile in bottom panel of Fig. 2).

\subsubsection{Transverse position-velocity diagrams}

We show in Fig. 7 transverse position-velocity maps reconstructed by integrating spectra within a 0.2 wide pseudo slit positioned perpendicular to the jet axis at two different distances from the star along the jet axis. These two position-velocity diagrams illustrate the transverse structure of the jet. Close to the source (at $d=00^{\prime} 4$ ), where the LVC component still marginally contributes, the emission on the jet axis peaks at intermediate velocities $\left(-60 \mathrm{~km} \mathrm{~s}^{-1}\right)$ and is marginally resolved transversally. At $d=11^{\prime \prime} 2$, the emission is more resolved transversally and the radial velocities peak at $-80 \mathrm{~km} \mathrm{~s}^{-1}$ towards the jet axis, consistent with the HVC component. In both cases, radial velocities decrease by $20 \mathrm{~km} \mathrm{~s}^{-1}$ in the external parts of the jet at transverse distances $+/-0 .{ }^{\prime} 6$.

\subsubsection{Search for transverse velocity shifts}

Transverse velocity gradients, indicative of possible rotation within the jet body, have been previously reported for 5 TTS microjets (Bacciotti et al. 2002; Woitas et al. 2005; Coffey et al. 2004, 2007).
The principle of the measurement of rotation is to search for radial velocity differences between pairs of spectra emitted at two symmetrical transverse offsets $\pm d_{\perp}$ on either side of the jet axis. In an axisymmetric flow, these velocity differences $\Delta V=$ $V\left(+d_{\perp}\right)-V\left(-d_{\perp}\right)$ would be related to the rotation velocity by $V_{\phi}\left(d_{\perp}\right)=\Delta V /(2 \sin i)$, where $i$ is the inclination of the jet axis to the line of sight, provided $d_{\perp}$ is larger than the telescope PSF and on the order of the jet emission radius, to minimize convolution and projection effects (Pesenti et al. 2004).

The RY Tau transverse position-velocity diagrams presented in Fig. 7 do show a skewness in the outer contours indicative of transverse velocity gradients. Velocity shifts between two spectra can be measured either by the difference of the centroid velocities (determined by fitting a Gaussian profile to each line) or directly by cross-correlating the two profiles. We apply both methods to compute transverse velocity shifts at various transverse distances to the jet axis $\left(d_{\perp}\right)$ and distances from the central source along the jet axis $\left(d_{/ /}\right)$. We first averaged lenslets over boxes of width 0 . $^{\prime} 4$ along the jet and 0.2 across the jet, in order to increase the signal to noise ratio and to decrease the random wavelength calibration uncertainty (typically with 3-4 lenslets per box, the latter is down to $1 \sigma \sim 2.5 \mathrm{~km} \mathrm{~s}^{-1}$ ). The results are shown in Fig. 8 for $d_{\perp}=0$. .'2 and $d_{\perp}=0$.' 6 . Computed velocity shifts range between $-10 \mathrm{~km} \mathrm{~s}^{-1}$ and $+10 \mathrm{~km} \mathrm{~s}^{-1}$ for $d_{\perp}=0.2$, and between $-35 \mathrm{~km} \mathrm{~s}^{-1}$ and $+15 \mathrm{~km} \mathrm{~s}^{-1}$ for $d_{\perp}=0{ }^{\prime} 6$, and show no clear trend with distance along the jet axis.

The random errors due to noise associated with our transverse velocity shift measurements are assessed through a MonteCarlo study detailed in Appendix A. We show that the Gaussian fitting method is more accurate, and that the uncertainties associated with our velocity shifts measurements are well represented by the empirical formula $\sigma_{\Delta V}=90 / S N R \mathrm{~km} \mathrm{~s}^{-1}$, as expected theoretically for our typical line width (Porter et al. 2004). Based on the above formula, we thus expect a $1 \sigma$ uncertainty in velocity shifts derived from Gaussian fits of $\leq 4 \mathrm{~km} \mathrm{~s}^{-1}$ for peak $S N R \geq 20$, and $\leq 8.5 \mathrm{~km} \mathrm{~s}^{-1}$ for peak $S N R \geq 10$ (significantly larger than wavelength calibration errors). Of course these estimates are only valid as long as the observed profiles do not depart too much from a Gaussian profile, which is our case in the blueshifted jet of RY Tau. Otherwise, the cross-correlation technique should be preferred for measuring velocity shifts and the corresponding uncertainties will be larger (see Appendix A). For completeness, both the Gaussian centroid and the correlation method are shown here, and they give identical results.

The $3 \sigma$ error-bars derived from the profile $S N R$ using the above empirical formula from our Monte-Carlo study are plotted in Fig. 8, for each transverse velocity difference. The detected velocity shifts at $d_{\perp}= \pm 0.2$ are all compatible with zero (at the $3 \sigma$ level), as expected from beam-convolution effects. Indeed, for transverse distances significantly smaller than the spatial PSF, contributions to rotation signatures from receding and approaching flow lines should cancel out in the beam (Pesenti et al. 2004).

For transverse distances of the order of the jet width and larger than our spatial resolution $\left(d_{\perp}=0\right.$.' 6$)$ where rotation signatures should be optimized (Pesenti et al. 2004), the detected velocity shifts are also compatible everywhere with zero except marginally at two distances along the jet axis: $d_{/ /}=0 .{ }^{\prime} 4$ and $d_{/ /}=1$.'2 where we detect at a $2.5-3 \sigma$ level transverse velocity shifts of $\Delta V=V\left(d_{\mathrm{NE}}\right)-V\left(d_{\mathrm{SW}}\right)$ of $+15.4 \pm 5.9 \mathrm{~km} \mathrm{~s}^{-1}$ and $-19.6 \pm 4.6 \mathrm{~km} \mathrm{~s}^{-1}$ respectively. These transverse asymmetries in velocities are clearly visible on the two transverse position-velocity diagrams shown in Fig. 7, which correspond to these same two distances from the source. We discuss in 


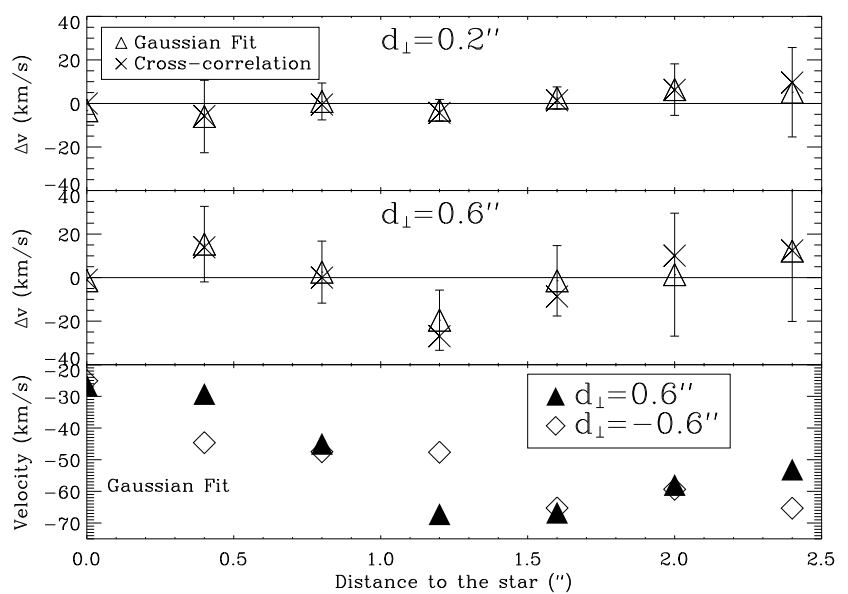

Fig. 8. Top and middle panels: velocity shifts between symmetric spectra taken at \pm 0.2 (top panel) and \pm 0. ' 6 (middle panel) on either side of the jet axis, as a function of distance along the blue jet. Velocity shifts are measured by one-component Gaussian fitting (triangles) and crosscorrelation (crosses). The $3 \sigma$ error-bar deduced from the profile peak $S N R$ is plotted. Bottom panel: centroid velocities (from Gaussian fits) measured on both sides of the jet axis at transverse offsets $d_{\perp}= \pm 0$ '. 6 , as a function of distance to the star. Positive transverse offsets (filled triangles) correspond to the north-east side of the blueshifted jet. In all panels, lenslets are averaged inside boxes of width $0 .{ }^{\prime} 4$ along the jet and 0.2 across the jet.

Sect. 4.3 whether or not these two marginally detected velocity shifts could trace signatures of rotation within the jet body.

\subsection{Mass-loss rate}

We estimate the mass-loss rate in the blueshifted jet from the [O I] line luminosities using two different methods described in detail by Hartigan et al. (1995), hereafter HEG95, and Cabrit (2002). The first method assumes volume emission over the entire elementary aperture under uniform plasma conditions $\left(n_{\mathrm{e}}\right.$, $\left.T_{\mathrm{e}}, x_{\mathrm{e}}=n_{\mathrm{e}} / n_{\mathrm{H}}\right)$. The mass-loss rate is then expressed as:

$\dot{M}_{J}=M_{\mathrm{TOT}} V_{\perp} / l_{\perp}$

where $V_{\perp}$ is the tangential flow velocity, $l_{\perp}$ is the projected size of the aperture on the plane of the sky along the jet axis, and $M_{\mathrm{TOT}}$ is the total gas mass inside the aperture given by:

$M_{\mathrm{TOT}}=9.61 \times 10^{-6}\left(\frac{1}{1-x_{\mathrm{e}}}\right)\left(1+\frac{n_{\mathrm{cr}}}{n_{\mathrm{e}}}\right)\left(\frac{L_{[\mathrm{OI}] 6300}}{L_{\odot}}\right) M_{\odot}$

assuming $T_{\mathrm{e}}=8200 \mathrm{~K}$, with a corresponding line critical density $n_{\mathrm{cr}}=1.97 \times 10^{6} \mathrm{~cm}^{-3}$ (HEG95). The term $\left(1-x_{\mathrm{e}}\right)$ is added here to account for ionised oxygen, assuming that $\mathrm{O}^{+} / \mathrm{O}=x_{\mathrm{e}}$ due to resonant charge exchange with hydrogen (Cabrit 2002).

The second method assumes that [OI] line emission within each elementary aperture arises from shock fronts. The massloss rate is then expressed as a function of the shock velocity as:

$\dot{M}_{J}=6.616 \times 10^{-4}\left(\frac{V_{\text {jet }}}{V_{\text {shock }}}\right)\left(\frac{L_{[\mathrm{O} I] 6300}}{L_{\odot}}\right)\left(\frac{\cos \theta}{N_{\text {shock }}}\right) M_{\odot} \mathrm{yr}^{-1}$

where $V_{\text {jet }}$ and $V_{\text {shock }}$ are the jet speed and shock speed respectively, $N_{\text {shock }}$ is the number of shocks within the elementary aperture and $\theta$ is the angle between the normal to the shock front and the jet axis. These last two parameters are not present in the original equation of HEG95, and are added to account for multiple shocks in the beam and for the fact that an oblique shock intercepts a surface of $1 / \cos \theta$ times the jet cross-section (Cabrit 2002).

By comparing the above two methods applied to stellar jets observed with both small and large apertures, Cabrit (2002) concluded that on average $\cos \theta / N_{\text {shock }} \simeq 1$ per $100 \mathrm{AU}$ of jet length. We will thus assume a value of 1 here for this ratio (our flux apertures have $l_{\perp}=0.4^{\prime \prime}=60 \mathrm{AU}$ along the jet, see below). We will adopt a jet speed of $V_{\text {jet }}=165 \mathrm{~km} \mathrm{~s}^{-1}$, and a tangential velocity $V_{\perp}=150 \mathrm{~km} \mathrm{~s}^{-1}$, corresponding to a blue jet inclination to the line of sight of $65^{\circ}$ (see Sect. 4.1 for estimates of the RY Tau jet inclination).

Since our present dataset of RY Tau only includes the [O I] line, values of $x_{\mathrm{e}}, n_{\mathrm{e}}$, and $V_{\text {shock }}$ cannot be inferred directly from [S II] and [N II] line ratios, as done previously in our OASIS study of the DG Tau microjet (Lavalley-Fouquet et al. 2000) and we have to rely on estimates. We note that low shock velocities are expected from the non-detection of [N II] emission in RY Tau (Hartigan et al. 1995; Hirth et al. 1997). Indeed, planar shock models predict a sharp decrease of emissivity for this line for shock velocities below $30 \mathrm{~km} \mathrm{~s}^{-1}$ (Hartigan et al. 1994). We may thus assume in Eq. (3) that $V_{\text {shock }}$ is in the range 20 to $50 \mathrm{~km} \mathrm{~s}^{-1}$. For $n_{\mathrm{e}}$ and $x_{\mathrm{e}}$ in Eq. (2), we will adopt typical parameters inferred from high-angular resolution observations of the inner regions of the DG Tau, RW Aur, Th 28 and HH 30 microjets (Lavalley-Fouquet et al. 2000; Dougados et al. 2002; Bacciotti \& Eislöffel 1999; Hartigan \& Morse 2007). We thus assume an ionisation fraction $x_{\mathrm{e}}=10 \%$ and an electronic density decreasing with distance to the central source as $n_{\mathrm{e}}=5 \times 10^{3}\left(1^{\prime \prime} / \mathrm{r}\right) \mathrm{cm}^{-3}$ for $d \geq 60 \mathrm{AU}$, and flattening to a constant value of $3 \times 10^{4} \mathrm{~cm}^{-3}$ inside 60 AU (Hartigan \& Morse 2007).

We estimate the blueshifted HVC jet [O I] luminosities at different distances along the jet axis by integrating observed surface brightnesses over the 3 velocity channels centered at -117 , -76 and $-35 \mathrm{~km} \mathrm{~s}^{-1}$ (i.e. a total velocity range from -137.5 to $-14.5 \mathrm{~km} \mathrm{~s}^{-1}$ taking into account the channel width). We now include the spectral channel centered at $-35 \mathrm{~km} \mathrm{~s}^{-1}$ since it always contains a significant fraction of the total HVC flux, due to our moderate spectral resolution (see Fig. 2). The emission is summed in the raw maps over rectangular apertures of full longitudinal and transverse widths of $0.4=l_{\perp}$ and $11^{\prime \prime} 6=d_{\perp}$ respectively. The chosen longitudinal width provides a sampling along the jet axis similar to our spatial PSF, while the full transverse width ensures that we include all of the jet emission over our field of view (measured transverse $F W H M \leq 0$.' 8 in our raw maps for distances along the jet axis $\leq 2$ '.). The derived HVC [O I] luminosities as a function of projected distance along the jet axis are plotted in Fig. 9. We observe a steep decrease in brightness with distance from the source, reaching two orders of magnitude at 2.5". However, within 0.' 6 from the central source, the measured [O I] luminosities are strongly contaminated by the strong low-velocity component, as indicated by centroid velocities lower than $-70 \mathrm{~km} \mathrm{~s}^{-1}$ (see Fig. 6). Beyond distances of $2^{\prime \prime}$, part of the transverse aperture falls outside the OASIS observations field of view. Distances between 0.' 6 and 1". 8 from the star (hereafter denoted as the HVC-dominated region) thus give the best estimate of the HVC jet luminosity.

We also plot in Fig. 9 our estimates of mass-loss rates using the methods of volume (Eq. (2)) and shock (Eq. (3)) emission. Since we assume for simplicity a constant value of shock speed and $\cos \theta / N_{\text {shock }}=1$, and since $V_{\text {jet }}$ also remains constant within $10 \%$ along the jet, the mass-flux rate we derive from the shock method is exactly proportional to the [O I] luminosity (see Eq. (3)) and thus follows the same steep decrease with distance 


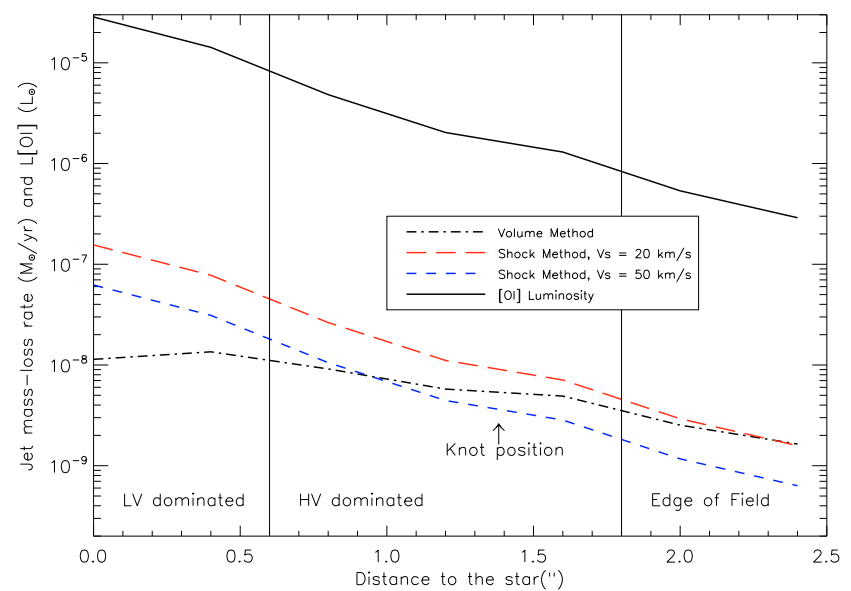

Fig. 9. Mass-loss rate in the RY Tau blueshifted jet as a function of projected distance, derived from the [O I] line luminosity with two different assumptions (see text): volume emission (dot-dashed line) and shock layer with shock speed $20 \mathrm{~km} \mathrm{~s}^{-1}$ (red long-dashed line) or $50 \mathrm{~km} \mathrm{~s}^{-1}$ (blue short-dashed line). The two methods are in good agreement in the region dominated by the HV component. The [O I] line luminosity, integrated over apertures of $0.4 \times 11^{\prime \prime} 6$ and velocity interval -137 to $-15 \mathrm{~km} \mathrm{~s}^{-1}$ is plotted as a full line.

to the central star. In particular, it drops by a factor of 5 from the $d=0.8^{\prime \prime}$ to the $d=1.6^{\prime \prime}$ apertures covering the HVC-dominated region. Although a real variation in $\dot{M}_{J}$ of this magnitude cannot be completely ruled out a priori over a time span of 4 yrs (crossing time of the HVC-dominated region), we suspect that this drop is mainly an artefact of our simplifying assumptions in the shock method. Our argument is that all TTS microjets images at sub-arcsecond resolution so far show a strongly decreasing [O I] jet luminosity over their inner $200 \mathrm{AU}$; If this decrease were proportional to a jet mass-flux variation, one would expect to also encounter stars with rising jet brightness over the same distance scales, whereas none have been seen. Therefore, the jet mass-flux is probably not strictly proportional to the jet luminosity over the jet length, and the factor 5 decline obtained by the shock method for a constant $V_{\text {shock }}$ and $N_{\text {shock }}$ is an upper limit to the true variation in $\dot{M}_{J}$ (e.g., $V_{\text {shock }}$ and $N_{\text {shock }}$ could easily decrease with distance from the star in a time-variable jet, causing a luminosity decline even for a constant mass-flux; see Eq. (3)).

In support of this conclusion, we note that the volume method gives values that are much more uniform along the jet, because the decline in [O I] luminosity is now compensated for by the drop of $n_{\mathrm{e}}$ (see Eq. (2)). Across the HVC-dominated region, values of the mass-loss rate derived by this method change by only a factor 2 , and are well bracketed by the shock method for low to intermediate shock speeds $\left(20-50 \mathrm{~km} \mathrm{~s}^{-1}\right)$. We thus take the average volume method mass-loss rate over distances of $0.6-11^{\prime \prime} 8$ from the star of $6.6 \times 10^{-9} M_{\odot} \mathrm{yr}^{-1}$ as our best estimate of the mean HVC mass-loss rate. The uncertainty on the jet velocity (see below), and on the typical electronic densities at projected distances 100-150 AU, suggests an uncertainty of at most a factor of 4 either way, consistent with the good agreement with the shock methods noted above. The mass-loss rate in the RY Tau blueshifted jet therefore is between 0.16 and $2.6 \times 10^{-8} M_{\odot} \mathrm{yr}^{-1}$. Combining with the range of disc mass accretion rates determined by Calvet et al. (2004) of 6.4-9.1 $\times 10^{-8} M_{\odot} \mathrm{yr}^{-1}$, we derive an ejection to accretion rate ratio (one sided) of $\dot{M}_{J} / \dot{M}_{\text {acc }}$ between 0.02 and 0.4 , with a most probable value of 0.085 .
Our best mass-flux estimate for the RY Tau blueshifted jet is 4 times higher than the value of $1.6 \times 10^{-9} M_{\odot} \mathrm{yr}^{-1}$ previously derived by HEG95 from long-slit spectroscopy, despite using the same volume method (Eq. (2)) and same transverse jet speed $\left(150 \mathrm{~km} \mathrm{~s}^{-1}\right)$. The main origin of this difference lies in the fact that our observations are spatially resolved while the ones of HEG95 were not. First, HEG95 assumed that their integrated [O I] HVC luminosity uniformly filled a large region of length $l_{\perp}=1^{\prime \prime}$. 25 originating at the star position. However, our $[\mathrm{O} \mathrm{I}]$ maps show that most of the $[\mathrm{O} \mathrm{I}]$ luminosity originates from a compact component located much closer to the source. Indeed, in our central aperture at $d=0^{\prime \prime}$ we measure a similar HVC [O I] luminosity as HEG95 did $\left(2.85 \times 10^{-5} L_{\odot}\right.$ here vs. $3.4 \times 10^{-5} L_{\odot}$ in HEG95), but with a diaphragm 3 times smaller $\left(l_{\perp}=0\right.$.' 4 here versus 1 1'.25 in HEG95). Furthermore, we noted above that our HVC luminosity is overestimated in the central regions, due to contamination by the compact LVC, so that the best estimate of the RY Tau jet HVC mass-loss rate is in fact not obtained at the source, but at projected distances along the jet between 0 . $^{\prime} 6$ to $11^{\prime \prime} 8$. Although we observe at these distances an order of magnitude lower [O I] luminosities than that derived by HEG95, we now have 16 times lower $n_{\mathrm{e}}$ values (adopting the representative $n_{\mathrm{e}}$ observed on these spatial scales in other resolved microjets) and again 3 times lower $l_{\perp}$, resulting in our best estimate mass-loss rate being, in the end, higher by a factor 4 than the value derived by HEG95. This example illustrates the key importance of spatially resolved spectro-imaging observations to derive more accurate mass-flux rates in $\mathrm{T}$ Tauri microjets.

\section{Discussion}

\subsection{Jet inclination angle and flow kinematics}

The RY Tau system inclination to the line of sight is currently poorly constrained. Kitamura et al. (2002) derive a best fit disc axis inclination angle of $43.5^{\circ} \pm 5$ from simultaneously fitting marginally resolved $2 \mathrm{~mm}$ dust continuum emission maps and the spectral energy distribution. On the other hand, Muzerolle et al. (2003) derive an inner disc rim inclination to the line of sight of $86^{\circ} \pm 10^{\circ}$ from modelling of the near-infrared spectral energy distribution (from 2 to $5 \mu \mathrm{m}$ ). Recently, Schegerer et al. (2008) constrained the disc inclination axis to the line of sight to be less than $70^{\circ}$ from fitting both the spectral energy distribution and $N$ band visibilities obtained with MIDI at the VLTI. Here we reexamine constraints on the system inclination in an attempt to better estimate the true deprojected jet speed.

The observed variation of line-of-sight velocities with position along the RY Tau jet, $\Delta V_{\text {rad }} \simeq 10 \% V_{\text {rad }}$, implies a typical shock speed $\simeq 10 \% V_{\text {jet }}$. Shock velocities in excess of $30 \mathrm{~km} \mathrm{~s}^{-1}$ at distances $\leq 1^{\prime \prime}$ from the source appear unlikely, since strong [N II] has never been observed in RY Tau (Hirth et al. 1997; Hartigan et al. 1995), while planar shock J-type models predict a sharp increase of [N II $] 6584 \AA$ emission above $V_{\mathrm{s}}=$ $30 \mathrm{~km} \mathrm{~s}^{-1}$. Hence the constraint derived above on shock velocities implies a true jet flow velocity lower than $300 \mathrm{~km}^{-1}$. With $V_{\text {rad }} \simeq 70 \mathrm{~km} \mathrm{~s}^{-1}$, this would correspond to a maximum inclination to the line of sight of $76.5^{\circ}$. This maximum inclination is compatible with the fact that no dark lane is clearly visible in optical/near-IR images of the system (St-Onge \& Bastien 2008).

We now derive an additional constraint on the minimum system inclination required to reproduce the photo-polarimetric behaviour of RY Tau reported by Petrov et al. (1999). As pointed out by these authors, the behaviour of RY Tau is reminiscent of 
that observed in UX Ori type stars, in particular the increase of linear degree of polarization when the system is fainter. Natta \& Whitney (2000) model the optical photometric and polarimetric variability of UX Ors with partial occultation of the photosphere by circumstellar dust clouds, resulting in a relative increase of (polarised) scattered radiation from the surrounding circumstellar disc. In particular, these authors compute the degree of linear polarisation expected at minimum light as a function of the optical depth of the occulting screens and of the inclination of the disc to the line of sight. In RY Tau, the intrinsic linear polarisation in the $V$ band increased from $0.7 \%$ at high brightness to $1.7 \% \pm 0.1 \%$ at minimum brightness $(\Delta \mathrm{mag}=1 \mathrm{mag}$; Petrov et al. 1999). According to the models computed by Natta \& Whitney (2000) this behaviour indicates an inclination of the disc axis to the line of sight $\geq 45^{\circ}$. The models are computed for a $2 M_{\odot}$ central star with effective temperature $T_{\text {eff }}=9000 \mathrm{~K}$ and luminosity $L_{\star}=50 L_{\odot}$, i.e. of similar mass as RY Tau, but of higher effective temperature and luminosity $\left(T_{\text {eff }}=5945 \mathrm{~K}\right.$ and $L_{\star}=9.6 \pm 1.5 L_{\odot}$ is estimated for RY Tau by Calvet et al. 2004). The model predictions are mostly sensitive to the disc flaring parameter $h / r$, ranging between 0.01 and 0.03 . For a passive irradiated thin disc, $T \propto L_{\star}^{1 / 4}$ so that $h / r \propto L_{\star}^{1 / 8} M_{\star}^{-1 / 2}$ and the difference of a factor 5 in stellar luminosities amounts to a $20 \%$ difference in $h / r$ only. The conclusion on the minimum RY Tau inclination angle of $45^{\circ}$ therefore appears quite robust.

Our conservative lower and upper limits to the jet inclination of $45^{\circ}$ and $76.5^{\circ}$ are compatible, within the errors, with the determinations of both Kitamura et al. (2002) and Muzerolle et al. (2003) but do not allow us to discriminate between the two. Within best available constraints, we will therefore assume a jet inclination angle to the line of sight within this range, implying a deprojected flow velocity between 100 and $300 \mathrm{~km} \mathrm{~s}^{-1}$ with a most probable value of $165 \mathrm{~km} \mathrm{~s}^{-1}$ (taking into account a random orientation of the jet axis in $3 \mathrm{D}$ space). This latter value was adopted to estimate the jet mass-flux rate.

We note that St-Onge \& Bastien (2008) estimate a similar proper motion of $165 \mathrm{~km} \mathrm{~s}^{-1}$ for their brightest $\mathrm{H} \alpha$ knot (Ha2), from comparison to archival HST data. If we identify our HVB [O I] knot at 1". 35 with one of their inner $\mathrm{H} \alpha$ knots, we would infer proper motions of $140 \mathrm{~km} \mathrm{~s}^{-1}$ (HaB knot) to $247 \mathrm{~km} \mathrm{~s}^{-1}$ ( $\mathrm{HaC}$ knot), again consistent with a moderate jet speed $<300 \mathrm{~km} \mathrm{~s}^{-1}$.

\subsection{Origin of the Hipparcos photocenter variation}

The jet position angle $\left(294^{\circ} \pm 1^{\circ}\right)$ is compatible with the position angle of the photocenter variation derived by Hipparcos observations $\left(304^{\circ} \pm 34^{\circ}\right)$, calling into question the proposed interpretation in terms of a close binary system (Bertout et al. 1999). One would expect close binaries to have their orbits coplanar with the disk and perpendicular to the jet. In an inclined system like RY Tau, the probability of catching the binary when it appears projected along the blueshifted jet axis would then be quite small. Furthermore, recent infrared interferometric measurements have failed to detect a close companion in RY Tau (Schegerer et al. 2008).

We investigate below whether the displacement of the photocenter seen by Hipparcos could be produced instead by line emission associated with the jet itself. A displacement of the photocenter in the direction of the blueshifted jet axis would result if the contrast between the extended jet and unresolved continuum photosphere varied during the Hipparcos observations (2.5 years between January 1990 and June 1992). Such a variation could be produced either by intrinsic jet variability due for example to knot ejections, or by variability in the photospheric continuum emission itself. The latter case seems to be favored in the RY Tau system, where the RY Tau photo-polarimetric behavior can be understood in terms of partial occultation episodes of the photosphere (Petrov et al. 1999).

We evaluate the total jet line flux expected in the Hipparcos photometric filter, covering the spectral range between $4000 \AA$ and $6500 \AA$, by considering the predictions from the planar shock models of Hartigan et al. (1994) and HEG95 with preshock densities ranging between $10^{6}$ and $10^{2} \mathrm{~cm}^{-3}$, pre-shock magnetic fields between 30 and $300 \mu \mathrm{G}$ and shock velocities ranging between 20 and $100 \mathrm{~km} \mathrm{~s}^{-1}$. The lines which could contribute significantly are: $\mathrm{H} \beta \lambda 4861 \AA$, [N I] $\lambda 5200 \AA$, [O I] $] \lambda$ 6300, $6363 \AA,[\mathrm{N} \mathrm{II}] \lambda \lambda 6548,6583 \AA, \mathrm{H} \alpha \lambda 6563 \AA$, and [S II] $\lambda \lambda 6716,6731 \AA$. We consider first a single shock front of shock velocity $V_{\mathrm{s}}$, located at distance $z$ from the source, and intercepting the total cross-section of the jet. The line emission can be expressed as:

$$
F(z)=\frac{\pi R(z)^{2}}{2 \pi D^{2}} \times f\left(V_{\mathrm{s}}(z), n_{\mathrm{H}}(z)\right)
$$

where $D$ is the distance from the source to the earth, $R(z)$ and $n_{\mathrm{H}}(z)$ respectively the jet transverse radius and pre-shock density at distance $z$ along the jet. The function $f$ gives the emitted flux per unit surface out of one face of the shock, and is taken from Hartigan et al. (1994) and HEG95. The function $f$ increases strongly with shock velocity and is proportional to $n_{\mathrm{H}}$, the preshock density. Its dependence on the pre-shock magnetic field is weak in the conditions considered. If we further assume a constant mass-loss rate along the jet, equal to $\pi R^{2} \mu n_{\mathrm{H}} V_{\text {jet }}$, the line flux becomes independent of $z$ and reduces to (for $D=140 \mathrm{pc}$ ):

$$
\begin{aligned}
F= & 2.1 \times 10^{-12} \times\left(\frac{\dot{M}_{J}}{10^{-8} M_{\odot} \mathrm{yr}^{-1}}\right) \times\left(\frac{165 \mathrm{~km} \mathrm{~s}^{-1}}{V_{\text {jet }}}\right) \\
& \times f\left(V_{\mathrm{s}}, n_{\mathrm{H}}=10^{4} \mathrm{~cm}^{-3}\right) \mathrm{erg} \mathrm{s}^{-1} \mathrm{~cm}^{-2}
\end{aligned}
$$

The function $f\left(V_{\mathrm{s}}, n_{\mathrm{H}}=10^{4} \mathrm{~cm}^{-3}\right)$ increases from $10^{-2}$ for shock velocities $V_{\mathrm{s}}=30 \mathrm{~km} \mathrm{~s}^{-1}$ to 0.2 for $V_{\mathrm{s}}=100 \mathrm{~km} \mathrm{~s}^{-1}$. Hence, the total optical line flux emitted by one single shock front is expected to be $\leq 2.1 \times 10^{-14} \mathrm{erg} \mathrm{s}^{-1} \mathrm{~cm}^{-2}$ for shock velocities $\leq 30 \mathrm{~km} \mathrm{~s}^{-1}, \leq 4.2 \times 10^{-13} \mathrm{erg} \mathrm{s}^{-1} \mathrm{~cm}^{-2}$ for shock velocities $\leq 100 \mathrm{~km} \mathrm{~s}^{-1}$.

The maximum photocenter displacement will occur at minimum photospheric brightness. During Hipparcos observations RY Tau $V$ band magnitudes varied between 9.7 and 11.05 . We thus estimate the continuum flux at minimum brightness in the Hipparcos photometric filter $(F W H M \simeq 2000 \AA)$ to be $F_{\text {cont }} \simeq$ $3 \times 10^{-10} \mathrm{erg} \mathrm{s}^{-1} \mathrm{~cm}^{-2}$. The maximum contrast between line emission from a single shock front and photospheric continuum emission is therefore expected to be $6.7 \times 10^{-5}$ for $V_{\mathrm{s}} \leq 30 \mathrm{~km} \mathrm{~s}^{-1}$ and $1.4 \times 10^{-3}$ for $V_{\mathrm{s}} \leq 100 \mathrm{~km} \mathrm{~s}^{-1}$.

To produce the maximum displacement of the photocenter of 19.5 mas observed by Hipparcos would thus require a shock front located at a distance from the star $\geq 300^{\prime \prime}$ for shock velocities $\leq 30 \mathrm{~km} \mathrm{~s}^{-1}$ and $14^{\prime \prime}$ for shock velocities $\leq 100 \mathrm{~km} \mathrm{~s}^{-1}$. But this is well outside the primary beam of Hipparcos (of diameter $30^{\prime \prime}$ but with a strong attenuation starting at $5^{\prime \prime}$ from center). Alternatively, more than one shock front may contribute within the beam of Hipparcos observations. The photocenter displacement produced by $n$ shock fronts equally spaced along the 
jet within a radius $R$, i.e. with separations $\Delta x=R / n$, can be written as:

$x_{\text {phot }}=R \times \frac{n+1}{2} \times \frac{F}{F_{\text {cont }}}$.

To produce a photocenter displacement of 19.5 mas would then require $\simeq 5$ shock fronts located within $5^{\prime \prime}$ of the central source for shock velocities $\simeq 100 \mathrm{~km} \mathrm{~s}^{-1}$, and an unrealistically large number of shock fronts $(\geq 100)$ for shock velocities $\leq 30 \mathrm{~km} \mathrm{~s}^{-1}$.

Knot separations $\simeq 1^{\prime \prime}$ are compatible with previous observations of the inner regions of TTS microjets (Dougados et al. 2000; Bacciotti et al. 2000; López-Martín et al. 2003; Woitas et al. 2005). Thus predictions of jet line emission from multiple shocks could marginally account for the observed Hipparcos photocenter displacement provided shock velocities are $\simeq 100 \mathrm{~km} \mathrm{~s}^{-1}$. This however seems inconsistent with the nondetection of [N II] emission in RY Tau. Progress on this issue will require more accurate determinations of shock velocities through line ratio observations in the RY Tau microjet.

Another possible source of photocenter displacement along the jet axis is bright scattered optical light from the surface of the circumstellar disc. At intermediate inclinations for the RY Tau system, the image of the disc in scattered light will be dominated by the blue lobe (see Natta \& Whitney 2000 for an example). Occultation of the photosphere will enhance the contrast between scattered and direct stellar light, resulting in a displacement of the photocenter perpendicular to the disc plane, towards the blueshifted jet axis. The maximum degree of linear polarisation of $1.7 \%$ at minimum photospheric brightness (Petrov et al. 1999) gives a lower limit to the ratio of scattered to direct light during occultation. Indeed due to polarisation cancelling in a centro-symmetric scattering pattern, the true ratio of scattered to direct light can be much higher than the integrated polarisation. This interpretation should be checked with detailed disc image modelling.

From the fact that the photocenter displacement seen by Hipparcos lies along the jet axis, we thus conclude that a binary companion may not be the most relevant explanation. Our analysis above shows however that line emission from shocks in the jet is probably not bright enough to produce the observed photocenter displacement in the Hipparcos broad photometric filter. The most likely origin thus appears to be scattering off the surface of the disc seen at intermediate inclinations, combined with variable partial occultation of the central photosphere. This interpretation should however be checked with a detailed study of the observed variation of the Hipparcos photocenter with time. In particular, we predict a clear anti-correlation between system brightness and photocenter displacement.

\subsection{Transverse structure and rotation signatures}

We discuss below whether the transverse velocity shifts marginally detected at distances along the jet of $d_{/ /}=0 .{ }^{\prime} 4$ and $1^{\prime \prime} .2$ from the star and transverse distances $d_{\perp}= \pm 0 . ' 6$ from the jet axis could be due to rotation within the jet body. The $2.5 \sigma$ detection at $d_{/ /}=0$. ' 4 is doubtful, since the inferred sense of rotation would be opposite to the disc rotation sense inferred from the velocity gradient observed in the millimetric CO observations of Koerner \& Sargent (1995): from blueshifted in the northeast to redshifted in the south-west along $\mathrm{PA} \simeq 21^{\circ}$. In contrast, the $3 \sigma$ detection at $d_{/ /}=1$ '. 2 would indicate a sense of rotation in the jet in agreement with the disc rotation sense. The detection of jet rotation at this position and not at others could reflect the fact that both the SNR and the HVC contribution are maximized there: closer in, photospheric subtraction residuals are important and the low-velocity component contributes significantly, while further out, the signal to noise ratio rapidly decreases. However, interpretation as a rotation signature remains questionable due to the marginal $3 \sigma$ level and to the fact that the detection happens to be very close to the jet knot detected in the HVB map. We plot in Fig. 5 as white boxes the two regions of the jet whose summed line profiles produce the detected transverse velocity shift at 1.2 . The detected velocity asymmetry could also be caused by asymmetries in the wings of a bow-shock peaked at the position of the HVB knot, at 1". 35 from the star (asterisk symbol in the figure). Higher angular resolution observations that better resolve the jet transverse structure are required to settle the origin of this velocity asymmetry.

\subsection{Comparison with cTTS microjets}

We analyze here the derived properties of the RY Tau microjet in the light of previous studies of T Tauri small scale jets. We compare in Fig. 4 the RY Tau blueshifted jet widths as a function of projected distance to the central source with previous studies of TTS microjets. Both the jet widths and opening angle appear similar to those derived on similar spatial scales for the other TTs microjets, suggesting similar collimation scale and properties.

The full range of centroid flow velocities observed on spatial scales of a few 100 AUs for the DG Tau (Pyo et al. 2003; Lavalley et al. 1997), RW Aur (Pyo et al. 2006), HL Tau (Pyo et al. 2006), HH 30 (Hartigan \& Morse 2007) and CW Tau (Hartigan et al. 2004) microjets, where inclination angles are well constrained, is $130-300 \mathrm{~km} \mathrm{~s}^{-1}$ with an average value of $225 \pm 55 \mathrm{~km} \mathrm{~s}^{-1}$. Our possible interval of $100-300 \mathrm{~km} \mathrm{~s}^{-1}$ for the deprojected terminal velocity in the RY Tau microjet (Sect. 4.1) thus falls in the same range as previously investigated microjets from lower mass central sources.

Finally, our estimate for the (one-sided) ejection to accretion rate $\dot{M}_{J} / \dot{M}_{\text {acc }}$ ratio, between 0.02 and 0.4 with a best value of 0.085 , is also compatible with the average ratio $\simeq 0.1$ for lower mass cTTS sources found by Cabrit (2007) using jet mass-fluxes from HEG95 and revised accretion rates from Gullbring et al. (1998).

The overall properties (collimation, terminal centroid velocities, ejection to accretion rate ratio) of the RY Tau microjet therefore appear quantitatively similar to those of the microjets from lower mass cTTS investigated so far.

\subsection{Implications for jet launching models}

The remarkably similar collimation, terminal speed, and ejection efficiency of the RY Tau microjet compared to lower mass cTTs gives for the first time a firm support to the common belief that the jet launching mechanism is probably universal over a broad range of stellar masses. We examine below the implications of the derived RY Tau jet parameters for the three classes of steady state jet launching magneto-centrifugal models currently discussed in the context of cTTS microjets and for which detailed observational predictions are available (see Ferreira et al. 2006; Cabrit 2007, for detailed reviews).

Magnetized stellar wind models have been developped to account for the origin of collimated mass-loss in young stars, and to explain their spin-equilibrium despite active accretion (Sauty \& Tsinganos 1994; Matt \& Pudritz 2008). Since CTTS rotate at a small fraction of their break-up velocity (e.g. 15\% in RY Tau), centrifugal acceleration from the stellar surface is insufficient 
to overcome the gravitational pull of the star, and a significant amount of energy must be deposited by pressure gradients at the base of the flow. This additional source of energy would presumably originate from the accretion process, e.g. in the form of enthalpy (hot wind) or Alfven-wave pressure (cool wind).

A recent analysis of UV emission lines in RY Tau by Gómez de Castro \& Verdugo (2007) does indicate the presence of a hot stellar wind at $\simeq 6 \times 10^{4} \mathrm{~K}$ in this star. However, with the parameters derived by these authors for the UV line emitting region (emitting volume $\left(0.35 R_{\odot}\right)^{3}$, total density $\simeq 10^{10} \mathrm{~cm}^{-3}$ and flow velocity $\simeq 150 \mathrm{~km} \mathrm{~s}^{-1}$ ), we estimate a mass-loss rate for the high-temperature UV emitting wind of $\simeq 10^{-11} M_{\odot} \mathrm{yr}^{-1}$. This is $2-3$ orders of magnitude lower than the mass-loss rate in the optical jet. Thus, the hot stellar wind component does not contribute significantly to the bulk of the jet mass-loss seen at optical wavelengths, and enthalpy is not the main jet driving mechanism.

Pressure gradients from Alfvén waves allow one to drive cooler stellar winds (Decampli 1981), but the wave energy needed to power the observed jet mass flux is unrealistically large. Neglecting centrifugal acceleration, the fraction of accretion luminosity that needs to be transferred to the flow is $\epsilon_{\infty} \simeq \beta \dot{M}_{j} / \dot{M}_{\mathrm{a}}$ where $\dot{M}_{j}$ is the mass-flux in one jet and $\beta=2+V_{\mathrm{jet}}^{2} / V_{\mathrm{K}, \star}^{2}$ parametrizes the amount of specific energy transferred to the flow by pressure gradients (Ferreira et al. 2006). In RY Tau, the estimated range of jet terminal speed is $100-300 \mathrm{~km} \mathrm{~s}^{-1}$ and the Keplerian speed at the stellar surface $V_{\mathrm{K}, \star}=350 \mathrm{~km} \mathrm{~s}^{-1}$ (using $M_{*}=2 M_{\odot}$ and $R_{*}=3 R_{\odot}$; Calvet et al. 2004), yielding $\beta \simeq 2-3$ and $\epsilon_{\infty} \simeq 20 \%-30 \%$ for the onesided ejection/accretion ratio of 0.1 . Full MHD stellar wind solutions including magnetocentrifugal effects yield a similar result, with $\epsilon_{\infty} \simeq 20 \%-40 \%$ for a two-sided ejection/accretion ratio of $0.15-0.2$ (Matt \& Pudritz 2008). If only 20\% of the Alfvén wave power is actually transferred to the flow, as the calculations of Decampli (1981) suggest, the total power in waves would have to be comparable to the accretion luminosity, which seems unrealistic.

We now examine the possibility that the jet originates from the inner regions of the circumstellar disc (X-wind or extended disc wind). The expression of the terminal poloidal flow velocity for a given streamline originating from a launching radius $r_{0}$ in the disc is given by (Blandford \& Payne 1982):

$V_{\mathrm{p}}^{\infty}=\left(G M_{*} / r_{0}\right)^{1 / 2} \sqrt{2 \lambda-3}$

where $\lambda=\left(r_{A} / r_{0}\right)^{2}$ is the magnetic lever arm parameter.

In the framework of the X-wind model developed by Shu and collaborators, all the streamlines originate from the co-rotation radius in the disc with $\lambda$ values ranging between 3 and 6 (Shang et al. 1998), with most of the mass flux having $\lambda \simeq 3$. With the RY Tau stellar parameters $M_{*}=2 M_{\odot}, R_{*}=3 R_{\odot}$, and $v \sin i=$ $52 \mathrm{~km} \mathrm{~s}^{-1}$, we estimate a co-rotation radius of $0.05 \sin i^{2 / 3} \mathrm{AU}$. Thus, the range of terminal poloidal velocities predicted by the $\mathrm{X}$-wind model in the case of the RY Tau microjet would be $\simeq 310-540(\sin i)^{-1 / 3} \mathrm{~km} \mathrm{~s}^{-1}$, with the jet line emission sharply peaked at the lower value (see predicted PV diagrams in Shang et al. 1998). The [O I] velocity centroid of $70 \mathrm{~km} \mathrm{~s}^{-1}$ in the RY Tau jet would then imply an inclination of the jet axis to the line of sight of $77^{\circ}$, at the upper limit of current observational constraints (see Sect. 4.1).

In the radially self-similar disc wind solutions first introduced by Blandford \& Payne (1982), mass-loss originates from an extended range of radii in the disc, and the magnetic lever arm parameter $\lambda$ is constant over all streamlines. Self-consistent calculations including the accretion disk dynamics show that superAlfvénic solutions exist for $\lambda$ between 2 and 100, depending on the toroidal field and heating at the disk surface (Ferreira 1997; Casse \& Ferreira 2000). The two-sided ejection to accretion rate ratio is tightly related to $\lambda$ and is given by $\frac{1}{2(\lambda-1)} \ln \left(r_{\text {out }} / r_{\text {in }}\right)$ where $r_{\text {out }}$ and $r_{\text {in }}$ are the inner and outer radii of the region in the disc where the jet originates. As shown in Ferreira et al. (2006), these solutions reproduce the global properties (collimation, terminal poloidal velocities, rotation signatures, ejection to accretion rate ratio) of previously studied cTTs microjets for $\lambda$ values in the range $2-15$ and $r_{\text {out }} \leq$ a few AUs. The global derived RY Tau jet parameters, being in the same range as previously investigated cTTS microjets, will therefore be compatible with similar disc wind solutions. We note in particular that the transverse kinematical structure of the blueshifted jet, illustrated in Fig. 7, is reminiscent of the kinematical behavior of magnetocentrifugal disc winds where outer streamlines originating from larger launching radii are predicted to flow at lower velocities than inner streamlines.

Our current best estimates of the RY Tau microjet parameters thus appear more compatible with an origin from the inner regions of the disc than with a stellar wind. Obviously, an accurate determination of the jet parameters (inclination and densities) is required to constrain more precisely the wind kinematics and the ejection to accretion ratio, allowing a detailed comparison with model predictions.

\section{Conclusions}

Using the OASIS spectroimager at CFHT, we have studied with $0.2^{\prime \prime}-0.4^{\prime \prime}$ resolution the $2 \mathrm{D}$ morphology and kinematics of the inner $2^{\prime \prime}$ of the RY Tau microjet in the [O I] $\lambda 6300 \AA$ line. Our main conclusions are the following:

- The [O I] emission exhibits a bright compact low-velocity peak within 30 mas $=4 \mathrm{AU}$ of the star, and an extended highvelocity blueshifted jet at $\mathrm{PA}=294^{\circ} \pm 1^{\circ}$, with an emission knot at 1 '. 35 . The PA is consistent with the $\mathrm{H} \alpha$ jet imaged on larger scales by St-Onge \& Bastien (2008). The redshifted jet is faint and only marginally detected.

- We derive PSF-corrected jet widths of 30-40 AU and full opening angle of $5^{\circ}$ on spatial scales 20-280 AU. These collimation properties are similar to those of lower mass cTTS microjets previously imaged at subarcsecond resolution.

- The mean jet centroid radial velocity is $-70 \pm 5 \mathrm{~km} \mathrm{~s}^{-1}$. Best current constraints indicate an intermediate jet inclination to the line of sight (between $45^{\circ}$ and $77^{\circ}$ ) and true flow velocities between 100 and $300 \mathrm{~km} \mathrm{~s}^{-1}$, in the same range as microjets from lower mass cTTS. The most probable jet speed of $165 \mathrm{~km} \mathrm{~s}^{-1}$ agrees well with preliminary proper motion estimates.

- The transverse jet kinematic structure was studied in detail. A decrease of centroid velocity towards the jet edges is observed at all positions along the jet axis, as expected from MHD disk wind models. Asymmetries in velocity with respect to the jet axis, and their statistical errors, were also examined. A $3 \sigma$ transverse velocity shift is found near the emission knot at 1'.35 from the source, but our angular resolution is insufficient to distinguish between jet rotation and bowshock asymmetries.

- From the measured spatially resolved [O I] luminosity along the jet, we derive for the HVC an ejection rate 4 times higher than the previous determination by Hartigan et al. (1995). 
The one-sided ejection to accretion rate ratio is $\simeq 0.1$ (within a factor of 4 ), similar to its average value in lower mass cTTS (Cabrit 2007).

- The remarkably similar collimation, terminal speed, and ejection efficiency of the RY Tau microjet compared to lower mass cTTS gives firm support to the common belief that the jet launching mechanism may be universal over a broad range of stellar masses.

- The [O I] jet mass-flux exceeds by $2-3$ orders of magnitude the mass-flux in the hot stellar wind component discovered in UV lines by Gómez de Castro \& Verdugo (2007). This does not support thermal pressure as the main driving agent for the atomic jet. An Alfvén-wave driven, cool stellar wind is possible but energetically challenging.

- The PA we measure for the blueshifted jet is comparable to the PA of the photocenter variation detected by Hipparcos in RY Tau, calling into question its interpretation in terms of a binary system. We propose that the photocenter displacements may instead arise during UXOr-like occultation events, which enhance emission from the blueshifted jet and/or scattering cavity relative to the occulted photosphere. This would predict a clear anti-correlation between photocenter displacement and source brightness.

Acknowledgements. We are grateful to the anonymous referee for useful and constructive comments. V. Agra-Amboage, S. Cabrit, and P. Garcia wish to acknowledge financial and travel support through the Marie Curie Research Training Network JETSET (Jet Simulations, Experiments and Theory) under contract MRTN-CT-2004-005592. The work of P. Garcia was supported in part by the Fundação para a Ciencia e a Tecnologia through grant PTDC/CTEAST/65971/2006 from POTCI, with funds from the European program FEDER.

\section{Appendix A: Random errors on transverse velocity shift measurements}

In order to accurately estimate the uncertainties due to noise in our transverse velocity shift measurements, we perform a Monte-Carlo study adapted to our OASIS observational parameters. We simulate pairs of identical Gaussian spectra with total $F W H M 135 \mathrm{~km} \mathrm{~s}^{-1}$ or $175 \mathrm{~km} \mathrm{~s}^{-1}$, typical of our observations, and shift them by $\Delta V=10 \mathrm{~km} \mathrm{~s}^{-1}$ (comparable to the expected transverse velocity shifts due to rotation), using the same velocity sampling as in our data. We checked that the results shown below are in fact independent of the shift for $\Delta V \geq 10 \mathrm{~km} \mathrm{~s}^{-1}$. We then introduce random Gaussian noise typical of our observing parameters, i.e. a peak signal-to-noise ratio $(S N R)$ varying between 5 and 40. For each Monte-Carlo realisation of the noise statistics, we "measure" velocity shifts between the two spectra with the two different methods: difference between the velocity centroids derived from single component Gaussian fits, and cross-correlation between the pair of spectra. We perform 300 such Monte-Carlo realisations at a given $S N R$, and then compute the rms standard deviation of the 300 measured velocity shifts.

The result is shown in Fig. A.1 where we plot the standard deviation of the measured velocity shift as a function of initial peak signal to noise ratio for the 2 methods, and for the two values of input FWHM. This study demonstrates that the Gaussian fitting procedure gives the best accuracy compared to cross-correlation, and that the error increases steeply with decreasing peak $S N R$.

The theoretical accuracy on the centroid of a single component Gaussian profile of dispersion $\sigma_{\text {line }}$ dominated by photon noise and with infinite sampling is given by $\sigma_{\text {line }} / S N R$ (Porter et al. 2004) where the SNR is the signal over noise ratio at the line emission peak. Since we compute the difference

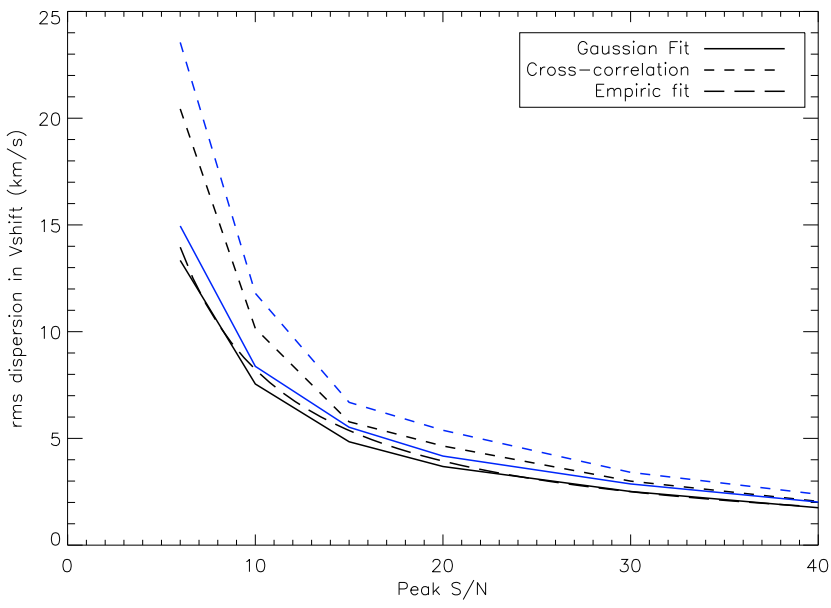

Fig. A.1. $1 \sigma$ error on the velocity shift between two spectra as a function of the signal-to-noise ratio $(S N R)$ at the line peak, as modelled from Monte-Carlo simulations. Two measurement methods are presented: cross-correlation of spectra (short-dashed curves) and centroid difference of Gaussian fits (solid curves). The input velocity shift is $10 \mathrm{~km} \mathrm{~s}^{-1}$, and the input profile $F W H M$ is $135 \mathrm{~km} \mathrm{~s}^{-1}$ (black curves) and $175 \mathrm{~km} \mathrm{~s}^{-1}$ (blue curves). The long dashed curve plots $\sigma_{\Delta V}=$ $90 / S N R \mathrm{~km} \mathrm{~s}^{-1}$, the expected theoretical error for infinite sampling (see text).

of two centroids, each affected by the same error, we would thus expect a $1 \sigma$ dispersion in the velocity shifts of $\sigma_{\Delta V} \simeq$ $\sqrt{2}(F W H M / 2.35) / S N R$ ranging between $82 / S N R \mathrm{~km} \mathrm{~s}^{-1}$ (for $F W H M=135 \mathrm{~km} \mathrm{~s}^{-1}$ ) and 104/SNR km s $\mathrm{km}^{-1}$ (for $F W H M=$ $175 \mathrm{~km} \mathrm{~s}^{-1}$ ). Our Monte-Carlo results in Fig. A.1 for the Gaussian method are well fitted by an empirical formula $\sigma_{\Delta V}=$ $90 / S N R \mathrm{~km} \mathrm{~s}^{-1}$, fully consistent with the theoretical expectations from Porter et al. (2004). We use this formula to compute $3 \sigma$ error bars for the transverse velocity shifts plotted in Fig. 8.

\section{References}

Bacciotti, F., \& Eislöffel, J. 1999, A\&A, 342, 717

Bacciotti, F., Mundt, R., Ray, T. P., et al. 2000, ApJ, 537, L49

Bacciotti, F., Ray, T. P., Mundt, R., Eislöffel, J., \& Solf, J. 2002, ApJ, 576, 222 Bacon, R., Emsellem, E., Combes, F., et al. 2001, A\&A, 371, 409 Basri, G., Martin, E. L., \& Bertout, C. 1991, A\&A, 252, 625 Bastien, P. 1982, A\&AS, 48, 153

Bertout, C., Robichon, N., \& Arenou, F. 1999, A\&A, 352, 574 Blandford, R. D., \& Payne, D. G. 1982, MNRAS, 199, 883 Cabrit, S. 2002, in EAS Pub. Ser. 3, ed. J. Bouvier, \& J.-P. Zahn, 147

Cabrit, S. 2007, in IAU Symp. 243, ed. J. Bouvier, \& I. Appenzeller, 203 Cabrit, S., Edwards, S., Strom, S. E., \& Strom, K. M. 1990, ApJ, 354, 687 Calvet, N., Muzerolle, J., Briceño, C., et al. 2004, AJ, 128, 1294

Casse, F., \& Ferreira, J. 2000, A\&A, 361, 1178

Coffey, D., Bacciotti, F., Woitas, J., Ray, T. P., \& Eislöffel, J. 2004, ApJ, 604, 758

Coffey, D., Bacciotti, F., Ray, T. P., Eislöffel, J., \& Woitas, J. 2007, ApJ, 663, 350

Corcoran, M., \& Ray, T. P. 1997, A\&A, 321, 189

Decampli, W. M. 1981, ApJ, 244, 124

Dougados, C., Cabrit, S., Lavalley, C., \& Ménard, F. 2000, A\&A, 357, L61

Dougados, C., Cabrit, S., \& Lavalley-Fouquet, C. 2002, in Rev. Mexicana Astron. Astrofis. Conf. Ser. 13, ed. W. J. Henney, W. Steffen, L. Binette, \& A. Raga, 43

Ferreira, J. 1997, A\&A, 319, 340

Ferreira, J., Pelletier, G., \& Appl, S. 2000, MNRAS, 312, 387

Ferreira, J., Dougados, C., \& Cabrit, S. 2006, A\&A, 453, 785

Garcia, P. J. V., Thiébaut, E., \& Bacon, R. 1999, A\&A, 346, 892

Gómez de Castro, A. I., \& Verdugo, E. 2007, ApJ, 654, L91

Goodson, A. P., Winglee, R. M., \& Boehm, K.-H. 1997, ApJ, 489, 199

Gullbring, E., Hartmann, L., Briceno, C., \& Calvet, N. 1998, ApJ, 492, 323 
Hartigan, P., \& Morse, J. 2007, ApJ, 660, 426

Hartigan, P., Morse, J. A., \& Raymond, J. 1994, ApJ, 436, 125

Hartigan, P., Edwards, S., \& Ghandour, L. 1995, ApJ, 452, 736

Hartigan, P., Edwards, S., \& Pierson, R. 2004, ApJ, 609, 261

Herbst, W., \& Stine, P. C. 1984, AJ, 89, 1716

Hirth, G. A., Mundt, R., \& Solf, J. 1997, A\&AS, 126, 437

Kitamura, Y., Momose, M., Yokogawa, S., et al. 2002, ApJ, 581, 357

Koerner, D. W., \& Sargent, A. I. 1995, AJ, 109, 2138

Lavalley, C., Cabrit, S., Dougados, C., Ferruit, P., \& Bacon, R. 1997, A\&A, 327, 671

Lavalley-Fouquet, C., Cabrit, S., \& Dougados, C. 2000, A\&A, 356, L41

López-Martín, L., Cabrit, S., \& Dougados, C. 2003, A\&A, 405, L1

Matt, S., \& Pudritz, R. E. 2008, ArXiv e-prints, 801

Mora, A., Merín, B., Solano, E., et al. 2001, A\&A, 378, 116

Muzerolle, J., Calvet, N., Hartmann, L., \& D’Alessio, P. 2003, ApJ, 597, L149

Natta, A., \& Whitney, B. A. 2000, A\&A, 364, 633
Pesenti, N., Dougados, C., Cabrit, S., et al. 2004, A\&A, 416, L9

Petrov, P. P., Zajtseva, G. V., Efimov, Y. S., et al. 1999, A\&A, 341, 553

Porter, J. M., Oudmaijer, R. D., \& Baines, D. 2004, A\&A, 428, 327

Pudritz, R. E., \& Norman, C. A. 1986, ApJ, 301, 571

Pyo, T.-S., Kobayashi, N., Hayashi, M., et al. 2003, ApJ, 590, 340

Pyo, T.-S., Hayashi, M., Kobayashi, N., et al. 2006, ApJ, 649, 836

Ray, T., Dougados, C., Bacciotti, F., Eislöffel, J., \& Chrysostomou, A. 2007, in

Protostars and Planets V, ed. B. Reipurth, D. Jewitt, \& K. Keil, 231

Sauty, C., \& Tsinganos, K. 1994, A\&A, 287, 893

Schegerer, A. A., Wolf, S., Ratzka, T., \& Leinert, C. 2008, A\&A, 478, 779

Shang, H., Shu, F. H., \& Glassgold, A. E. 1998, ApJ, 493, L91

Shu, F. H., Najita, J., Ostriker, E. C., \& Shang, H. 1995, ApJ, 455, L155

St-Onge, G., \& Bastien, P. 2008, ApJ, 674, 1032

Whelan, E. T., Ray, T. P., Bacciotti, F., et al. 2005, Nature, 435, 652

Woitas, J., Leinert, C., \& Köhler, R. 2001, A\&A, 376, 982

Woitas, J., Bacciotti, F., Ray, T. P., et al. 2005, A\&A, 432, 149 